\title{
Fission cross sections as a probe of fusion dynamics at high angular momentum
}

\author{
C. S. Palshetkar, ${ }^{*}$ D. J. Hinde, M. Dasgupta, E. Williams, K. Ramachandran, ${ }^{\dagger}$ I. P. Carter, ${ }^{\ddagger}$ \\ K. J. Cook, D. Y. Jeung, D. H. Luong, ${ }^{\S}$ S. D. McNeil, D. C. Rafferty, and A. Wakhle ${ }^{\mathbb{I}}$ \\ Department of Nuclear Physics, Research School of Physics and Engineering, The Australian National University, \\ Canberra, ACT 2601, Australia
}

(Received 23 March 2018; revised manuscript received 23 August 2018; published 4 October 2018)

\begin{abstract}
Background: Fusion of heavy nuclei requires energy dissipation to trap the system inside the capture barrier. At high angular momentum, the centrifugal potential causes the barrier radius to reduce, which may lead to energy dissipation outside the barrier, affecting the fusion angular momentum distributions and thus the capture cross sections.

Purpose: To investigate the sensitivity of fusion-fission cross sections as a probe of fusion dynamics at high angular momentum.

Method: Fission of the compound nucleus ${ }^{164} \mathrm{Yb}$ formed by three different fusion reactions, namely, ${ }^{16} \mathrm{O}+$ ${ }^{148} \mathrm{Sm},{ }^{28} \mathrm{Si}+{ }^{136} \mathrm{Ba}$, and ${ }^{40} \mathrm{Ca}+{ }^{124} \mathrm{Sn}$, was measured at four beam energies well above their respective capture barriers. Fission cross sections were extracted from the measured fission fragment angular distributions and compared with model calculations of fusion and subsequent fission. Fusion and evaporation residue cross sections available in the literature for the same or similar reactions were used to guide model calculations and obtain the fusion angular momentum distributions.

Results: The measured fission characteristics were found to be consistent with fusion-fission, as expected, justifying the use of the statistical model to calculate fission cross sections for each reaction. Significantly different fission cross-section predictions were obtained from calculations using angular momentum distributions corresponding to different coupling schemes and different diffuseness parameter of the nuclear potential. A large diffuseness parameter $(\gg 0.65 \mathrm{fm})$ of the nuclear potential was observed to give the best reproduction of both the experimental fusion as well as fission cross sections.

Conclusions: Experimental fission cross sections provide a stringent constraint to the fusion model calculations and thus prove to be a sensitive probe for understanding fusion dynamics at high angular momentum. This is shown in the present work by a simultaneous analysis of the fusion and fission cross sections for systems where fission cross sections form a small fraction of the fusion cross section and where noncompound nuclear processes are not a dominant competing channel. Observations from this work also suggest the requirement of evaporation residue as well as fission cross sections of higher precision than those generally available for drawing quantitative conclusions.
\end{abstract}

DOI: 10.1103/PhysRevC.98.044603

\section{INTRODUCTION}

The challenge of understanding fusion dynamics, particularly the relative competition between compound nuclear and noncompound nuclear processes, is a topic of continued

\footnotetext{
*Present address: Department of Nuclear and Atomic Physics, Tata Institute of Fundamental Research, Mumbai-400 005, India; asckj1@yahoo.co.in

${ }^{\dagger}$ Permanent address: Nuclear Physics Division, Bhabha Atomic Research Centre, Mumbai-400 085, India.

${ }^{\ddagger}$ Present address: CSIRO Sorting and Sensing, Mineral Resources, Building 67, ANSTO, Lucas Heights, Sydney, Australia.

${ }^{\S}$ Present address: Scandinavian Health Limited, Taiwan Branch, 136 Guosheng 2nd Street, Taoyuan District, Taoyuan City, Taiwan 330.

"Present address: Cyclotron Institute, Texas A\&M University, College Station, Texas 77843, USA.
}

interest. This is important, for example, in heavy element synthesis using fusion reactions [1,2]. Fusion results in the formation of a compound nucleus $(\mathrm{CN})$ once the interacting projectile and target nuclei overcome their mutual Coulomb barrier and kinetic energy is dissipated, leaving the system near the equilibrium deformation. With increasing projectiletarget charge product $\left(Z_{p} Z_{t}\right)$, angular momentum plays an increasingly important role in the reaction dynamics. The effect of angular momentum is generally accounted for by a centrifugal potential, arising from conservation of angular momentum. The increased contribution of this potential with increasing angular momentum causes a reduction in the barrier radius. Hence, for fusion to occur, the system must evolve further towards a compact shape before the (shallow) potential pocket is encountered. In such cases, energy dissipation, implicitly taken to occur only inside the pocket in fusion models, may also occur outside the fusion barrier [3]. This may result in very different angular momentum distributions of the $\mathrm{CN}$ and consequently the fusion cross sections. 
In the coupled-channels description of the fusion process, the fusion barrier and pocket arises from the sum of Coulomb, nuclear and centrifugal potentials, where the nuclear potential is generally parameterized by a Woods-Saxon form. The shape of the nuclear potential, that changes strongly according to its diffuseness, which is defined via the diffuseness parameter $a_{o}$, alters the position and depth of the pocket and thus the diffuseness parameter is a crucial input for fusion calculations. From a systematic study of fusion cross sections for a large number of reactions [3], large values of $a_{o}(\sim 0.7-1.4 \mathrm{fm})$ were found to be necessary to fit the excitation functions. In contrast, elastic and inelastic scattering data require $a_{o} \sim$ $0.65 \mathrm{fm}$ [4-7]. Using a diffuseness of this value results in the calculated fusion cross sections significantly larger than those measured. It has been suggested [3] that this discrepancy could be due to dynamical effects such as energy dissipation outside the barrier that are not accounted for in models of fusion. The large $a_{o}$ value required to fit the above-barrier fusion cross sections may be "mocking up" these dynamical effects. This is because a large $a_{o}$ leads to the fusion barrier occurring at a smaller radius, which in turn leads to reduced fusion cross sections at above-barrier energies compared to those obtained using $a_{o}=0.65 \mathrm{fm}$. Further, the larger value of $a_{o}$ results in a shallower potential pocket [8], which vanishes at lower angular momentum as compared to $a_{o}=0.65 \mathrm{fm}$ calculations. Thus, the fusion angular momentum distributions are cut off at lower $l$ values than for $a_{o}=0.65 \mathrm{fm}$, resulting in smaller cross sections.

The fission barrier and hence the fission probabilities depend on the angular momentum of the $\mathrm{CN}$ and hence the latter can provide important information on the evolution of reaction dynamics with angular momentum. For $\mathrm{CN}$ significantly lighter than $\mathrm{Pb}$ nuclei, fission is a significant decay mode only at high angular momentum $(l)$ and thus the fission cross sections are sensitive to the high $l$ tail of the distribution $[9,10]$. Sensitivity of fission cross sections to extreme variation of the shape of the fusion $l$ distribution (sharp-cutoff or diffuse distributions) has been previously demonstrated $[10,11]$ for reactions where fission is a small fraction of the fusion cross sections [10]. Thus, for such systems, fission cross sections should also be a sensitive probe to test fusion models at high angular momentum. With the aim of investigating this proposition in a quantitative way, a systematic study of the fusion-fission cross sections for the $\mathrm{CN}{ }^{164} \mathrm{Yb}$ formed through different entrance channels, namely, ${ }^{16} \mathrm{O}+{ }^{148} \mathrm{Sm},{ }^{28} \mathrm{Si}+{ }^{136} \mathrm{Ba}$, and ${ }^{40} \mathrm{Ca}+{ }^{124} \mathrm{Sn}$, has been carried out in the present work. The following points have been taken into consideration for this investigation: (i) The $\mathrm{CN}$ formed via the three different entrance channels is not expected to have competition from the noncompound nuclear process of quasifission. (ii) The measurements have been carried out at similar excitation energy range so as to be mostly sensitive to the angular momentum introduced in the fusion reactions. (iii) By choosing the same compound nucleus, fission calculations are constrained to use the same statistical model parameters for each reaction.

The paper is organized as follows. Experimental details are given in Sec. II followed by analysis and results in Sec. III. Extraction of the experimental fission fragment angular
TABLE I. Details of the target and ${ }^{12} \mathrm{C}$ backing thicknesses, fusion barrier energy $\left(V_{b}^{\text {lab }}\right)$ for each system, laboratory beam energies $\left(E_{\text {beam }}^{\text {lab }}\right)$, the energy loss corrected beam energies at the target center $\left(E_{\text {corr }}^{\text {lab }}\right)$, and the center of mass energies $\left(E_{\text {c.m. }}\right)$ in the present measurement.

\begin{tabular}{|c|c|c|c|c|c|c|c|}
\hline \multirow[t]{2}{*}{ Beam } & \multirow[t]{2}{*}{ Target } & \multicolumn{2}{|c|}{ Thickness } & \multirow{2}{*}{$\begin{array}{c}V_{b}^{\mathrm{lab}} \\
(\mathrm{MeV})\end{array}$} & \multirow{2}{*}{$\begin{array}{c}E_{\text {beam }}^{\mathrm{lab}} \\
(\mathrm{MeV})\end{array}$} & \multirow{2}{*}{$\begin{array}{c}E_{\mathrm{corr}}^{\mathrm{lab}} \\
(\mathrm{MeV})\end{array}$} & \multirow{2}{*}{$\begin{array}{c}E_{\mathrm{c} . \mathrm{m} .} \\
(\mathrm{MeV})\end{array}$} \\
\hline & & $\begin{array}{l}\text { Target } \\
\left(\frac{\mu \mathrm{g}}{\mathrm{cm}^{2}}\right)\end{array}$ & $\begin{array}{c}\text { Backing } \\
\left(\frac{\mu \mathrm{g}}{\mathrm{cm}^{2}}\right)\end{array}$ & & & & \\
\hline${ }^{16} \mathrm{O}$ & ${ }^{148} \mathrm{Sm}$ & 30 & 40 & $66.3^{\mathrm{a}}$ & $\begin{array}{r}81.95 \\
90.95 \\
100.00 \\
110.00\end{array}$ & $\begin{array}{r}81.91 \\
90.91 \\
99.96 \\
109.97\end{array}$ & $\begin{array}{c}73.92 \\
82.04 \\
90.21 \\
99.24\end{array}$ \\
\hline${ }^{28} \mathrm{Si}$ & ${ }^{136} \mathrm{Ba}$ & 40 & 12 & $114.5^{\mathrm{b}}$ & $\begin{array}{l}130.65 \\
140.49 \\
143.73 \\
151.38\end{array}$ & $\begin{array}{l}130.50 \\
140.34 \\
143.58 \\
151.24\end{array}$ & $\begin{array}{c}108.22 \\
116.38 \\
119.07 \\
125.42\end{array}$ \\
\hline${ }^{40} \mathrm{Ca}$ & ${ }^{124} \mathrm{Sn}$ & 17 & 12 & $157.0^{c}$ & $\begin{array}{l}166.81 \\
172.24 \\
184.25 \\
190.70\end{array}$ & $\begin{array}{l}166.65 \\
172.08 \\
184.09 \\
190.54\end{array}$ & $\begin{array}{c}126.00 \\
130.11 \\
139.19 \\
144.07\end{array}$ \\
\hline
\end{tabular}

a Taken from Ref. [21].

${ }^{\mathrm{b}}$ Estimated according to the procedure detailed in Sec. III B 2.

${ }^{\mathrm{c}}$ Taken from Ref. [27].

distributions and fission cross sections for the three systems is presented in Sec. III A. Details of the coupled-channels calculations, which reproduce the experimental fusion cross section and the $l$ distributions thus obtained are given in Sec. III B. Statistical model calculations for fission cross section predictions are described in Sec. IV. Here, a comparison of the calculated fusion and fission cross sections, which simultaneously reproduce the corresponding experimental values is discussed. A summary of the work along with the conclusions is given in Sec. V.

\section{EXPERIMENTAL DETAILS}

Fission fragment mass and angular distributions for the three systems were measured using the 14UD electrostatic tandem accelerator at the Heavy Ion Accelerator Facility at the Australian National University, Canberra. This provided pulsed beams of $\sim 1 \mathrm{~ns}$ FWHM to allow time-of-flight measurements. Details of the projectiles and targets used in the experiment are given in Table I. The CUBE spectrometer [12], consisting of two large area multiwire proportional counters (MWPCs), each having an active area of $27.9 \times 35.7 \mathrm{~cm}^{2}$ and giving an angular coverage in-plane of $75^{\circ}$, was employed for the detection of binary fission fragments in coincidence.

Figure 1 shows schematic diagrams of the detector setups used for these measurements. For the ${ }^{16} \mathrm{O}+{ }^{148} \mathrm{Sm}$ and ${ }^{28} \mathrm{Si}+$ ${ }^{136} \mathrm{Ba}$ measurements, one detector (MWPC 1) was placed at backward angles with the detector center at a polar angle of $\theta_{1}=135^{\circ}$ and azimuthal angle of $\phi_{1}=180^{\circ}$ giving an angular coverage of $\theta_{1}=95^{\circ}-170^{\circ}$ [Fig. 1(a)]. The other detector (MWPC 2) was placed at forward angles with respect to the beam axis, corresponding to the detector center at 
(a)

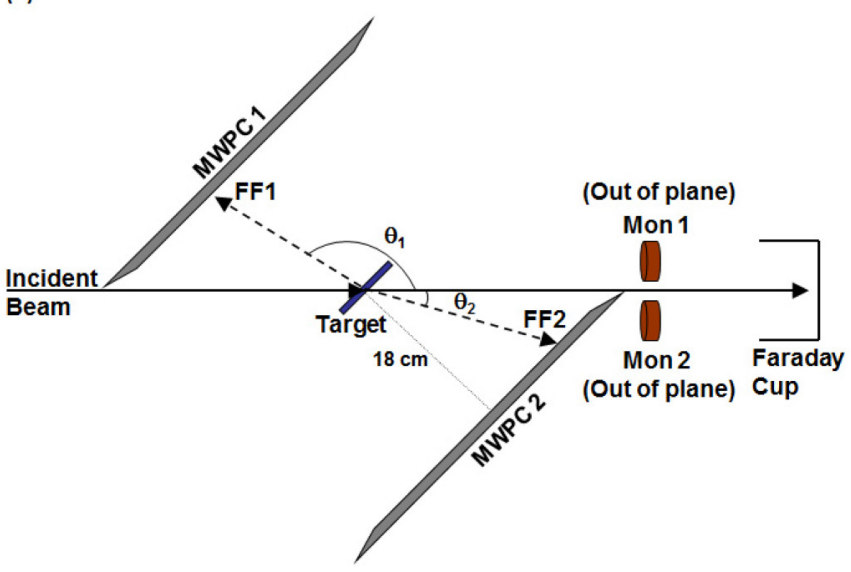

(b)

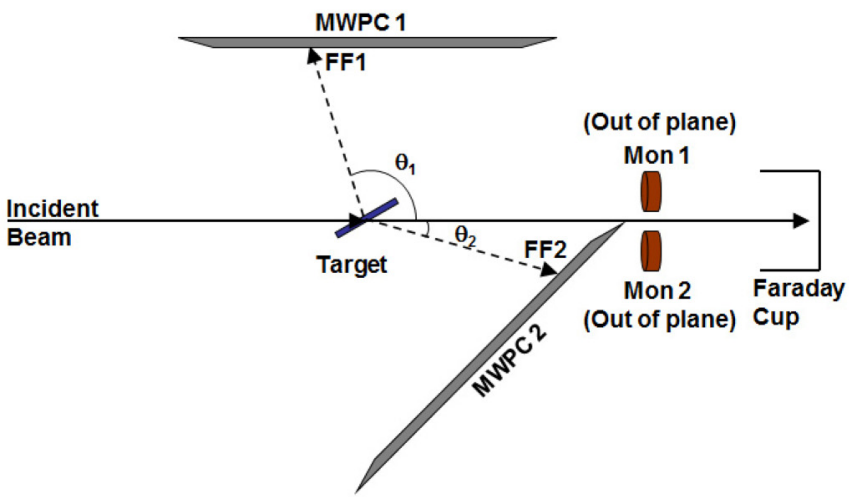

FIG. 1. Schematic diagram of the detector setup for the (a) ${ }^{16} \mathrm{O}+$ ${ }^{148} \mathrm{Sm},{ }^{28} \mathrm{Si}+{ }^{136} \mathrm{Ba}$ and $(\mathrm{b}){ }^{40} \mathrm{Ca}+{ }^{124} \mathrm{Sn}$ fission measurements.

$\theta_{2}=45^{\circ}$ and $\phi_{2}=0^{\circ}$ giving an angular coverage of $\theta_{2}=5^{\circ}$ to $80^{\circ}$. Two silicon surface barrier detectors were placed out of plane, at $\theta_{\text {mon }}=23^{\circ}$ and $\phi_{1}=90^{\circ}, \phi_{2}=270^{\circ}$ to detect the elastically scattered beam particles that were used for normalization. Due to the smaller folding angle of fission fragments for the ${ }^{40} \mathrm{Ca}+{ }^{124} \mathrm{Sn}$ system, the back detector (MWPC 1) was moved to more forward angles [Fig. 1(b)], with the detector centered at $\theta_{1}=90^{\circ}$ giving an angular coverage of $55^{\circ}-130^{\circ}$. The target was located $18 \mathrm{~cm}$ away from the central cathode foil of each MWPC.

Pulsed beams, with 107 ns separation bombarded the targets, whose normals were oriented at $45^{\circ}$ (for ${ }^{16} \mathrm{O}+{ }^{148} \mathrm{Sm}$ and ${ }^{28} \mathrm{Si}+{ }^{136} \mathrm{Ba}$ measurements) or $60^{\circ}$ (for ${ }^{40} \mathrm{Ca}+{ }^{124} \mathrm{Sn}$ measurement) with respect to the beam direction so as to avoid shadowing. Energy loss and time of flight of the fission fragments with respect to the beam pulse were recorded from the timing signals obtained from the central cathode foils of the MWPCs. Signals in MWPC 2 were recorded only when in coincidence with MWPC 1 in order to minimize the dead time of the data acquisition system. A pulser signal was inserted into the preamplifiers of the MWPCs and simultaneously counted in a scaler for dead time estimation. The fission and elastic events used for the cross-section determination were corrected for this dead time in the analysis. Position information of the fragments were recorded via delay line chips with each tap connected to individual anode wires (1 mm apart). The $X, Y$ position, energy loss and timing information for all detected particles was recorded event by event. All reactions were measured at four energies above their respective barriers (for values see Table I). Elastic scattering of ${ }^{28} \mathrm{Si}$ from ${ }^{197} \mathrm{Au}$ $\left(170 \mu \mathrm{g} / \mathrm{cm}^{2}\right.$ thick, self supporting) was also carried out at a laboratory energy of $74.3 \mathrm{MeV}$ for determining the solid angle in the case of the fission measurements for the first two systems. A similar measurement of scattering of ${ }^{40} \mathrm{Ca}$ from ${ }^{197} \mathrm{Au}$ target was carried out at a laboratory energy of 184.2 MeV for solid angle determination in the ${ }^{40} \mathrm{Ca}+{ }^{124} \mathrm{Sn}$ fission measurement. For the position calibration of each detector, events were recorded in singles mode where the whole active area of the detector is illuminated. Thus, from the known positions of the detector edges the position calibration was achieved. Time calibration was done by inputting pulses separated by $10 \mathrm{~ns}$ from a time calibrator and recording the resultant output pulses.

\section{ANALYSIS AND RESULTS}

The data analysis is described below in three parts: (i) extracting the experimental fission cross sections from the measured fission fragment angular distributions; (ii) coupledchannels calculations to get the best reproduction of the experimental fusion cross sections and obtain the $l$ distributions for each system for different coupling schemes; and (iii) statistical model calculations using the $l$ distributions obtained in step (ii) to predict fission cross sections for each system at the energies measured and their comparison with the experimental cross sections obtained in step (i).

\section{A. Experimental fission cross sections}

From the position and time-of-flight information, the emission angle of the detected fission fragments and their velocities were obtained. These velocities were then transformed into the ratio of fragment masses using the kinematic coincidence method (see Appendix of Ref. [12]). A clear selection of the fission events could be obtained from gates on the timeof-flight signals with respect to the beam in both detectors (see Fig. 2). Fission yields corresponding to $5^{\circ}$ bins in angle were extracted and were corrected for the detector solid angle to get the differential cross sections for each energy. The cross sections were converted from the laboratory frame to the center-of-mass frame assuming binary fission (which is a valid assumption for the systems studied in the present work) and by taking the average kinetic energy of the fragments given by the Viola systematics [13]. The experimental differential fission cross sections thus obtained are plotted in Fig. 3 for all three systems at the different energies measured. The uncertainties shown on the cross sections are statistical.

In order to determine the angle integrated fission cross sections from these differential cross sections, it is necessary to extrapolate the latter from the angular range covered in the experiment to $90^{\circ}$ and $180^{\circ}$. For this, the differential cross sections were fitted with transition state model calculations 

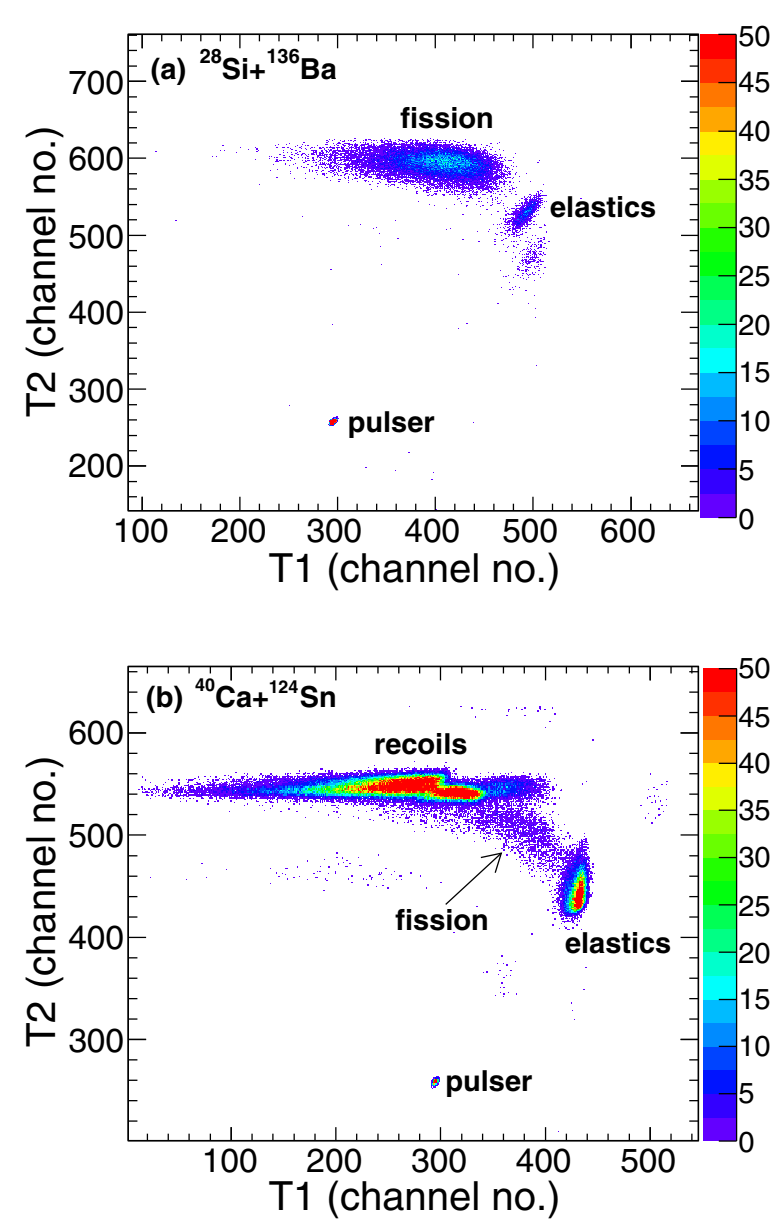

FIG. 2. Time of flight signals with respect to the beam pulse for MWPC 1 (T1) plotted against those from MWPC 2 (T2) recorded in the experiment for $(\mathrm{a})^{28} \mathrm{Si}+{ }^{136} \mathrm{Ba}$ and $(\mathrm{b}){ }^{40} \mathrm{Ca}+{ }^{124} \mathrm{Sn}$ reactions. using the following expression for spin zero nuclei [14]:

$$
W(\theta)=\sum_{J=0}^{\infty}(2 J+1) T_{J} \sum_{K=-J}^{J} \rho_{J}(K)\left|D_{0 K}^{J}\right|^{2} .
$$

Here, $T_{J}$ is the transmission coefficient for fusion of partial wave $J, K$ is the projection of the angular momentum on the symmetry axis, $\rho_{J}$ is the normalised distribution of final $K$ values given by [14]

$$
\begin{aligned}
\rho_{J}(K) & =\frac{\exp \left(-K^{2} / 2 K_{0}^{2}\right)}{\sum_{K=-J}^{J} \exp \left(-K^{2} / 2 K_{0}^{2}\right)} ; \quad K \leqslant J \\
& =0 ; \quad K>J,
\end{aligned}
$$

$D_{0 K}^{J}$ are the $\theta$-dependent symmetric top wave functions. $K_{0}$ in Eq. (2) is the standard deviation of the $K$ distribution. The model calculations were carried out by adjusting $K_{0}$ for a given $J$ value until a minimum $\chi^{2}$ fit to the experimental differential cross sections was obtained. The corresponding results are shown in Fig. 3 by lines for each energy. These were integrated over angle to get the total fission cross section at each energy (values given in Table II). The uncertainties on the total fission cross sections are mainly due to uncertainties in $K_{0}$. Due to the low statistics at the lowest energies measured for ${ }^{16} \mathrm{O}+{ }^{18} \mathrm{Sm}$ and ${ }^{28} \mathrm{Si}+{ }^{136} \mathrm{Ba}$ systems and limited angular range at all energies for the ${ }^{40} \mathrm{Ca}+{ }^{124} \mathrm{Sn}$ system, the anisotropy at these energies could not be determined uniquely. The $K_{0}$ values used in these cases (values given in Table II) resulted in an anisotropy of 3 for ${ }^{16} \mathrm{O}+{ }^{148} \mathrm{Sm}, 3.3$ for ${ }^{28} \mathrm{Si}+{ }^{136} \mathrm{Ba}$, and 3.5 for ${ }^{40} \mathrm{Ca}+{ }^{124} \mathrm{Sn}$ system, consistent with the trends at higher energies. A variation of $K_{0} \pm 5 \hbar$ gives a deviation of $5-20 \%$ in the fission cross sections for all three systems.

While investigating the concept of using fission cross sections to probe fusion at high angular momentum, an important
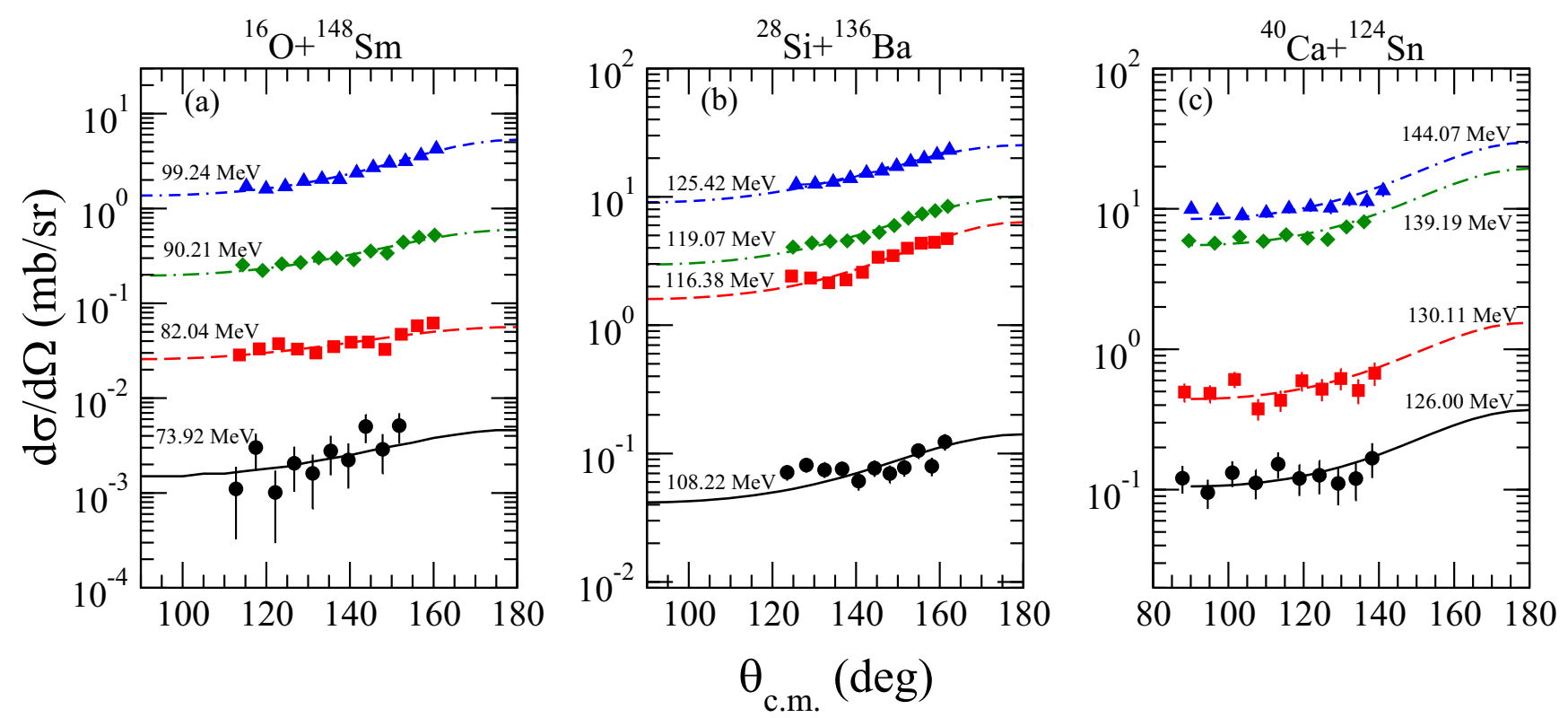

FIG. 3. Differential fission cross sections for (a) ${ }^{16} \mathrm{O}+{ }^{148} \mathrm{Sm}$, (b) ${ }^{28} \mathrm{Si}+{ }^{136} \mathrm{Ba}$, and (c) ${ }^{40} \mathrm{Ca}+{ }^{124} \mathrm{Sn}$ systems at the indicated center-ofmass energies. The lines represent the fits to the experimental data obtained from the transition state model calculations. 
TABLE II. Experimental fission cross sections $\left(\sigma_{\text {fis }}\right)$ extracted from the differential cross sections for the three systems studied in the present work. Values of the center-of-mass energies $\left(E_{\text {c.m. }}\right)$ and the excitation energy $\left(E^{*}\right)$ of the $\mathrm{CN}$, angular momentum $(J)$ and the standard deviation in the $K$ distribution $\left(K_{o}\right)$ are also given.

\begin{tabular}{lccccc}
\hline \hline System & $\begin{array}{c}E_{\text {c.m. }} \\
(\mathrm{MeV})\end{array}$ & $\begin{array}{c}E^{*} \\
(\mathrm{MeV})\end{array}$ & $\begin{array}{c}\sigma_{\text {fis }} \\
(\mathrm{mb})\end{array}$ & $\begin{array}{c}J \\
(\hbar)\end{array}$ & $\begin{array}{c}K_{0}{ }^{\mathrm{a}} \\
(\hbar)\end{array}$ \\
\hline \multirow{3}{*}{${ }^{16} \mathrm{O}+{ }^{148} \mathrm{Sm}$} & 73.92 & 50.87 & $0.013 \pm 0.008$ & 32.1 & 12.0 \\
& 90.21 & 59.00 & $0.21 \pm 0.01$ & 40.6 & $20.5 \pm 3.0$ \\
& 99.24 & 76.18 & $12.7 \pm 0.2$ & 53.8 & $6.6 \pm 1.1$ \\
& 108.22 & 58.86 & $0.38 \pm 0.06$ & 42 & 14.5 \\
${ }^{28} \mathrm{Si}+{ }^{136} \mathrm{Ba}$ & 116.38 & 67.02 & $14.9 \pm 0.3$ & 53 & $17.3 \pm 1.1$ \\
& 119.07 & 69.71 & $26.8 \pm 0.2$ & 56 & $19.4 \pm 0.4$ \\
& 125.42 & 76.06 & $78.5 \pm 0.6$ & 60 & $24.2 \pm 0.7$ \\
& 126.00 & 63.94 & $0.96 \pm 0.29$ & 48 & 16.1 \\
${ }^{40} \mathrm{Ca}+{ }^{124} \mathrm{Sn}$ & 130.11 & 68.04 & $4.0 \pm 0.7$ & 54 & 18.1 \\
& 139.19 & 77.13 & $50.2 \pm 3.5$ & 61 & 20.4 \\
& 144.07 & 82.00 & $77.2 \pm 6.4$ & 64 & 21.4 \\
\hline \hline
\end{tabular}

$\overline{\text { a See text for an explanation on } K_{o} \text { values for which uncertainties }}$ have not been given in the table.

factor that needs to be considered is the competition to the fusion-fission process from quasifission. For investigations such as the present in which the same $\mathrm{CN}$ is formed via different entrance channels, the presence of quasifission is undesirable. This is because the measured fission and quasifission cross sections would not be determined solely by the compound nuclear statistical decay parameters and the fusion angular momentum distribution. Since the aim of the present work is to investigate the sensitivity of the fission cross sections to fusion angular momentum distributions, systems exhibiting quasifission cannot be used.

A signature of the presence of quasifission can be clearly seen from the mass-angle distribution (MAD) where a dependence of the mass of the fission fragments on their emission angle is observed [15]. This was checked by evaluating the MAD for the ${ }^{40} \mathrm{Ca}+{ }^{124} \mathrm{Sn}$ system, having the largest $Z_{p} Z_{t}$, at the highest energy of $E_{\text {c.m. }}=144.07 \mathrm{MeV}$ measured in the present work, shown in Fig. 4. No signature of mass-angle correlation is observed from the MAD, suggesting that, as expected, quasifission is not significant for this system. It is thus not expected to be present for the other two lighter systems. Thus, the measured experimental fission cross sections (Table II) should be representative of fusion-fission, which is a requirement in this work.

\section{B. Coupled-channels calculations for fusion cross-section predictions}

To obtain a reliable measure of the sensitivity of fission cross sections to the fusion angular momentum, (i) a knowledge of the fusion $l$ distributions obtained from the measured fusion excitation functions and (ii) reliable prediction of the fission cross sections using these $l$ distribution are required. The two steps taken together ensure a consistent description of both fusion and fission cross sections. As detailed in the

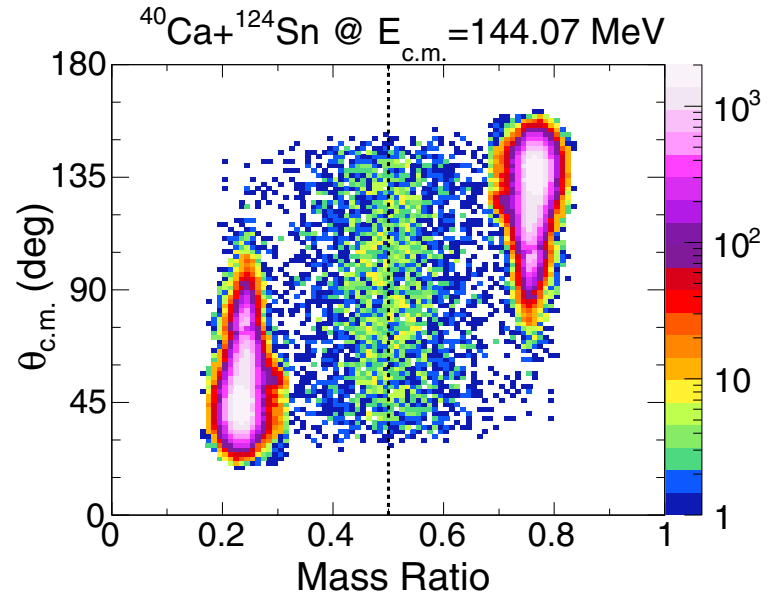

FIG. 4. Experimental MAD obtained for the ${ }^{40} \mathrm{Ca}+{ }^{124} \mathrm{Sn}$ system at $E_{\text {c.m. }}=144.07 \mathrm{MeV}$ in the present work. No correlation of mass ratio with angle is observed and all events are centered around mass ratio of 0.5 .

sections below, the first step was achieved by obtaining the best reproduction of the measured fusion excitation functions (corresponding to the three systems studied in the present work) available in the literature by the results of coupledchannels (CC) calculations done using the code CCFULL [16]. This allowed for obtaining realistic $l$ distributions for the three systems. These were used in the second step to obtain the fission cross-section predictions from statistical model calculations where the experimental fission cross sections were used to constrain the statistical model parameters.

The variable nuclear potential parameters in the $\mathrm{CC}$ calculations, the potential depth $V_{o}$, the radius parameter $r_{o}$, and the diffuseness parameter $a_{o}$, which largely govern the cross-section predictions, were constrained as follows: (i) Using the starting values of $V_{o}=200 \mathrm{MeV}, r_{o}=1.00 \mathrm{fm}$, and $a_{o}=1.0 \mathrm{fm}$ and without inclusion of coupling to inelastic or transfer channels, the parameters were varied to get a first estimate of $V_{o}, r_{o}$, and $a_{o}$ which best reproduce the experimental fusion cross sections at above barrier energies. (ii) Where good quality fusion excitation functions were available, the experimental barrier distributions were extracted by taking the second derivative of $\sigma_{\text {fus }} E_{\mathrm{c} . \mathrm{m}}$. with respect to $E_{\mathrm{c} . \mathrm{m}}$. (in steps of $2 \mathrm{MeV}$ ) using the three point difference method [17]. A comparison of these with the barrier distribution extracted from the $\mathrm{CC}$ cross-section predictions was done as a consistency check. The experimental average barrier values obtained from the distributions (see Table III for values) are consistent with those quoted in the literature $[21,28]$. (iii) The $2^{+}$and $3^{-}$vibrational states of the projectile and target were coupled one by one in the $\mathrm{CC}$ calculations and checked to see which states were important in determining the fusion cross sections and the experimental barrier distributions. Once a satisfactory reproduction of both was obtained, the coupling scheme was fixed and a final calculation was done to fine tune the potential parameters to be consistent with the average barrier energy but now with the couplings included. (iv) For the ${ }^{28} \mathrm{Si}+{ }^{136} \mathrm{Ba}$ and ${ }^{40} \mathrm{Ca}+{ }^{124} \mathrm{Sn}$ systems, the effect of transfer is expected to be 
TABLE III. Values of the experimental average fusion barrier (expt. $V_{b}^{\text {avg }}$ ) for the three systems along with the nuclear potential parameters $\left(V_{o}, r_{o}, a_{o}\right)$ used in the CC calculations for different coupling schemes described in the text for the standard diffuseness of $a_{o}=0.65 \mathrm{fm}$ and the larger diffuseness $\left(a_{o} \gg 0.65 \mathrm{fm}\right)$. The bare potential barrier energy $\left(V_{b}^{\text {bare }}\right)$ along with its corresponding radius $\left(R_{b}\right)$ values obtained in CCFULL are also given.

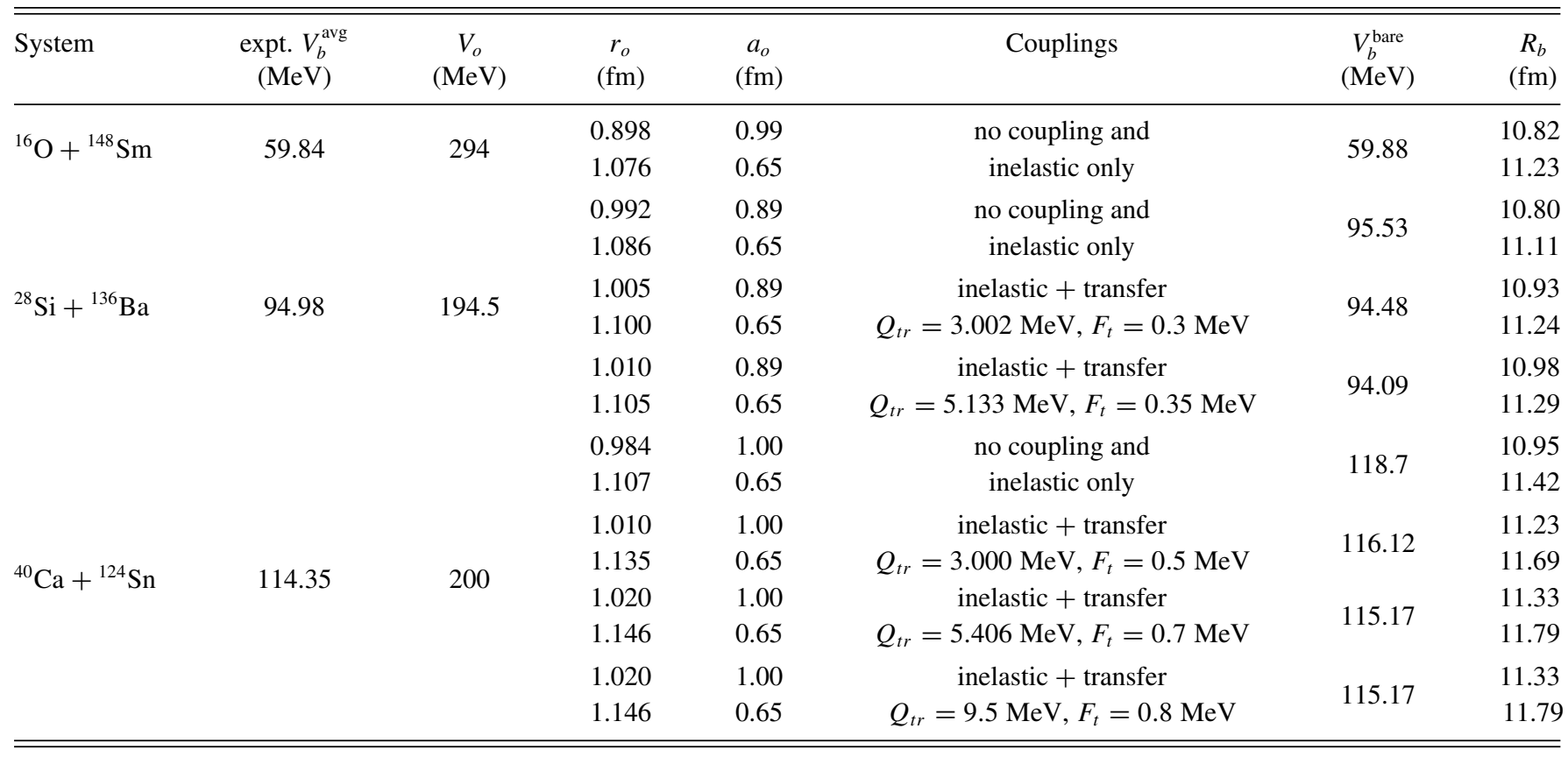

significant due to the positive $Q$ values for various neutron (n) transfer channels and hence was also included in the final calculations (described in the sections below for the two systems), especially to reproduce the near and below barrier fusion cross sections. The potential parameters obtained using the above procedure for different coupling schemes are given in Table III. We emphasize here that while the fits to the excitation functions were achieved here by including different couplings in the $\mathrm{CC}$ calculations, the couplings, especially to transfer channels, are a mechanism to reproduce the cross sections only and thus the coupling parameters should not be used for any other purpose.

For the purpose of comparison, $\mathrm{CC}$ calculations were also carried out using $a_{o}=0.65 \mathrm{fm}$. However, in these, a fit to the experimental fusion cross sections was not performed. Instead, using the same coupling scheme and the same $V_{o}$ as that obtained for the larger diffuseness, the value of $r_{o}$ was varied to reproduce the average barrier values given in Table III.

$$
\text { 1. }{ }^{16} \mathrm{O}+{ }^{148} \mathrm{Sm}
$$

Experimental fusion cross sections for the ${ }^{16} \mathrm{O}+{ }^{148} \mathrm{Sm}$ system, taken from Refs. [18-21], are plotted in Fig. 5 as filled circles. In the $\mathrm{CC}$ calculations, the ${ }^{16} \mathrm{O}$ projectile was taken as inert whereas rotational coupling with $\beta_{2}=0.18$ and $\beta_{4}=0.05$ was included for ${ }^{148} \mathrm{Sm}$ target, as in Ref. [21]. In addition, vibrational coupling of the $3^{-}$state of ${ }^{148} \mathrm{Sm}$ (for details see Table IV) was also included.

The results of the $\mathrm{CC}$ calculations, including the static deformations and inelastic coupling to the $3^{-}$state in ${ }^{148} \mathrm{Sm}$, are plotted in Fig. 5 for the diffuseness parameter of $a_{o}=$ $0.99 \mathrm{fm}$ (solid lines) and $a_{o}=0.65 \mathrm{fm}$ (dot-dashed lines). The energy of the average barrier is well matched using either of the diffuseness values as seen from Fig. 5(c).

Comparison of the $\mathrm{CC}$ results for the two $a_{o}$ values shows that, as expected [3] at above barrier energies, the larger $a_{o}$ value results in smaller fusion cross sections as compared to those for $a_{o}=0.65 \mathrm{fm}$ and gives an overall better reproduction of the experimental cross sections. The $l$ distributions obtained from the above calculations are plotted in Fig. 6 for the two $a_{o}$ values. It can be seen that as compared to $a_{o}=0.99 \mathrm{fm}$, the $l$ distributions for $a_{o}=0.65 \mathrm{fm}$ extend to large $l$ values, consistent with the fusion barrier radius being larger (and the potential pocket deeper). Inclusion of coupling extends the partial wave cross sections to higher $l$ values and it is clear that these would lead to very different fission cross sections as will be shown in Sec. IV.

$$
\text { 2. }{ }^{28} \mathrm{Si}+{ }^{136} \mathrm{Ba}
$$

Due to the unavailability of experimental fusion cross sections for ${ }^{28} \mathrm{Si}+{ }^{136} \mathrm{Ba}$ in the literature, those corresponding to ${ }^{28} \mathrm{Si}+{ }^{142} \mathrm{Ce}[24]$ were used as a reference for the $\mathrm{CC}$ calculations for the ${ }^{28} \mathrm{Si}+{ }^{136} \mathrm{Ba}$ system. To effectively take into account the geometrical effects for the two systems, the fusion cross sections as well as energies were scaled according to the procedure given in Ref. [25] as: $\sigma_{\text {fus }}^{\text {scaled }}=\sigma_{\text {fus }} /\left(A_{p}^{1 / 3}+A_{t}^{1 / 3}\right)^{2}$, $E_{\text {c.m. }}^{\text {scaled }}=E_{\text {c.m. }}\left(A_{p}^{1 / 3}+A_{t}^{1 / 3}\right) /\left(Z_{p} Z_{t}\right)$. The respective scaling factors of $0.0147,0.0102$ for ${ }^{28} \mathrm{Si}+{ }^{142} \mathrm{Ce}$ and $0.0149,0.0104$ for ${ }^{28} \mathrm{Si}+{ }^{136} \mathrm{Ba}$ were thus obtained. Using these, the scaled experimental fusion cross sections for the ${ }^{28} \mathrm{Si}+{ }^{142} \mathrm{Ce}$ system 


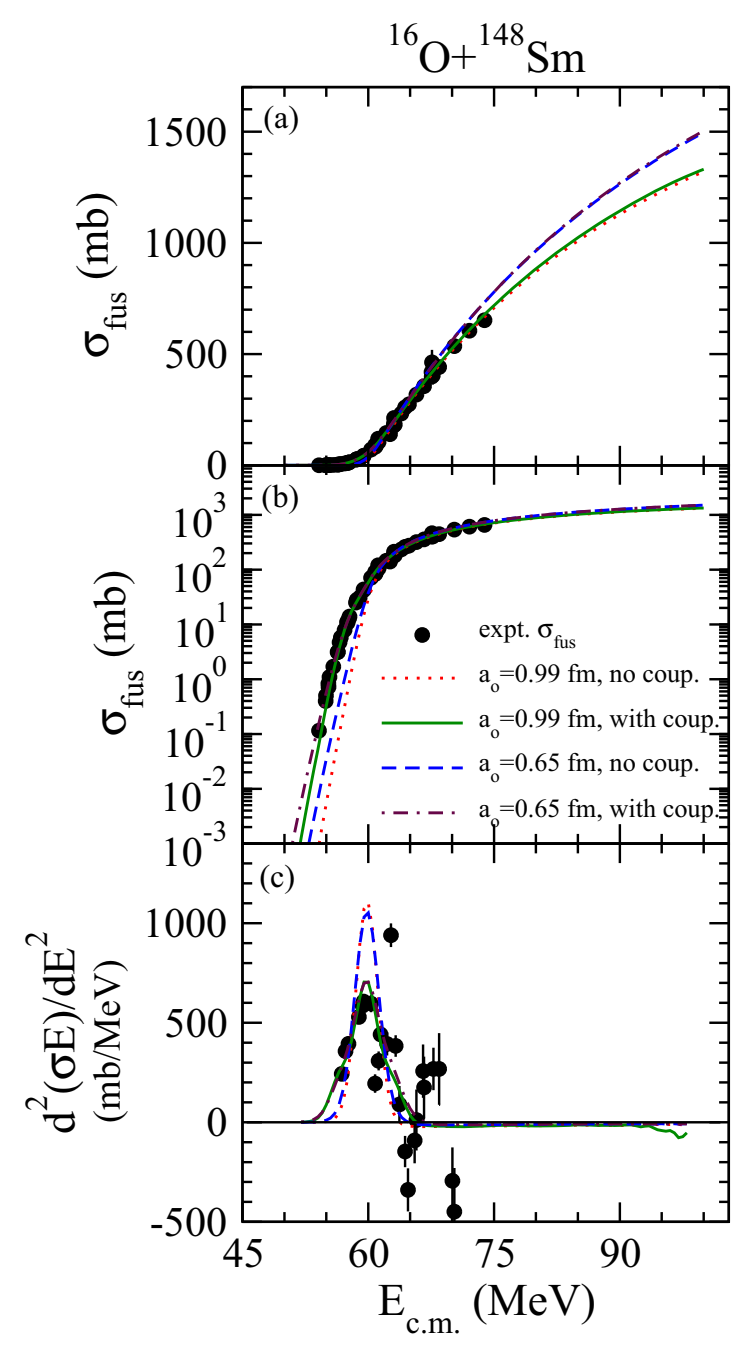

FIG. 5. Experimental fusion cross sections (filled circles) for the ${ }^{16} \mathrm{O}+{ }^{148} \mathrm{Sm}$ system taken from Refs. [18-21] plotted in (a) linear and (b) logarithmic scales. The extracted barrier distribution is shown by filled circles in (c). The dotted and dashed lines correspond to the calculations without any coupling for $a_{o}=0.99 \mathrm{fm}$ and 0.65 $\mathrm{fm}$, respectively. The solid lines show the $\mathrm{CC}$ calculations including static deformation and inelastic coupling to states in ${ }^{148} \mathrm{Sm}$ that best reproduce the experimental fusion cross sections while the dot-dashed lines are the CC results using the same coupling but for $a_{o}=0.65 \mathrm{fm}$. All lines in (c) have the same meaning as in (a), (b).

are plotted in Fig. 7 as filled circles whereas the scaled cross sections corresponding to the $\mathrm{CC}$ predictions for ${ }^{28} \mathrm{Si}+{ }^{136} \mathrm{Ba}$ described below are plotted by lines.

The average fusion barrier for the ${ }^{28} \mathrm{Si}+{ }^{136} \mathrm{Ba}$ system could in principle be obtained by scaling from the corresponding value for ${ }^{28} \mathrm{Si}+{ }^{142} \mathrm{Ce}$ system. However, due to fewer data points for the latter, the barrier distribution and thus the experimental average barrier could not be determined from this system. Hence, the average barrier for the ${ }^{28} \mathrm{Si}+{ }^{136} \mathrm{Ba}$ system was instead determined by scaling the experimental average barrier energy (103.9 MeV [3]) for the ${ }^{28} \mathrm{Si}+{ }^{144} \mathrm{Sm}$ system using a scaling factor of $Z_{p} Z_{t} /\left(A_{p}^{1 / 3}+A_{t}^{1 / 3}\right)$. An average barrier of $94.98 \mathrm{MeV}$ was obtained using this procedure.
TABLE IV. Values of the spin and parity $\left(J^{\pi}\right)$, excitation energy $\left(E_{x}\right)$, multipolarity $(\lambda)$ and reduced transition probabilities $[B(E \lambda \uparrow)]$ of the vibrational states used in the CC calculations for the different nuclei. All the $B(E 2 \uparrow)$ have been taken from Ref. [22] and $B(E 3 \uparrow)$ values from Ref. [23]. The $\beta_{2}$ values have been calculated from Eq. (2) in Ref. [22] with $R_{o}=1.2 A^{1 / 3} \mathrm{fm}$, while the $\beta_{3}$ values have been calculated from Eq. (3) in Ref. [23].

\begin{tabular}{llllll}
\hline \hline Nucleus & $J^{\pi}$ & $\begin{array}{c}E_{x} \\
(\mathrm{MeV})\end{array}$ & $\lambda$ & $\begin{array}{c}B(E \lambda \uparrow) \\
\left(e^{2} b^{\lambda}\right)\end{array}$ & $\beta_{\lambda}$ \\
\hline${ }^{148} \mathrm{Sm}$ & $3^{-}$ & 1.161 & 3 & 0.2911 & 0.1418 \\
${ }^{28} \mathrm{Si}$ & $2^{+}$ & 1.779 & 2 & 0.0326 & 0.4070 \\
${ }^{136} \mathrm{Ba}$ & $2^{+}$ & 0.818 & 2 & 0.410 & 0.1258 \\
& $3^{-}$ & 2.532 & 3 & 0.155 & 0.1253 \\
${ }^{40} \mathrm{Ca}$ & $3^{-}$ & 3.737 & 3 & 0.184 & 0.411 \\
${ }^{124} \mathrm{Sn}$ & $2^{+}$ & 1.132 & 2 & 0.1660 & 0.0953 \\
& $3^{-}$ & 2.603 & 3 & 0.073 & 0.1056 \\
\hline \hline
\end{tabular}

$\mathrm{CC}$ calculations were carried out for ${ }^{28} \mathrm{Si}+{ }^{136} \mathrm{Ba}$ by including the $2^{+}$state of ${ }^{28} \mathrm{Si}$ and the $2^{+}$and $3^{-}$states in ${ }^{136} \mathrm{Ba}$ in the vibrational coupling scheme (for details of all coupling parameters, see Table IV) and are plotted in Fig. 7 by the dashed lines. All calculations were done ensuring that the average barrier energy obtained from CC calculations matched that obtained from the scaling (i.e., $94.98 \mathrm{MeV}$ ). From the comparison of the scaled experimental fusion cross sections for ${ }^{28} \mathrm{Si}+{ }^{142} \mathrm{Ce}$ (filled circles) with the $\mathrm{CC}$ results for ${ }^{28} \mathrm{Si}+{ }^{136} \mathrm{Ba}$ corresponding to inelastic coupling (dashed line), it can be seen that inclusion of inelastic couplings alone cannot account for the experimental cross sections, especially at sub-barrier energies. Similar to the ${ }^{28} \mathrm{Si}+{ }^{142} \mathrm{Ce}$ system, this system has positive $Q$ values for different $n$-transfer

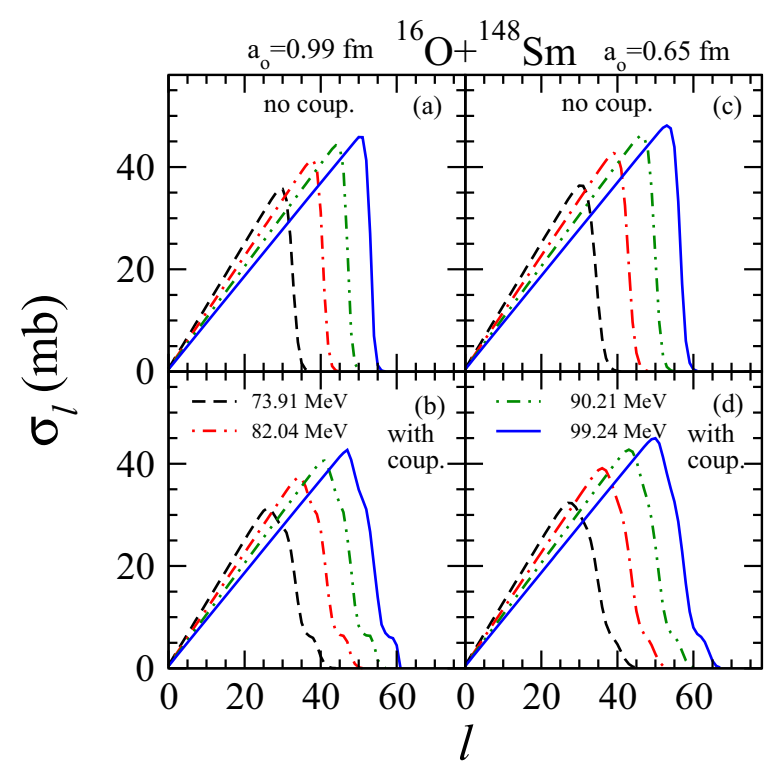

FIG. 6. Calculated $l$ distributions (a), (c) without coupling and (b), (d) with couplings (see text for details) for the two different diffuseness parameters. 
channels and thus their coupling is expected to influence the sub-barrier cross sections. Hence, in addition to the coupling to inelastic states, two different transfer couplings were tried: $Q_{t r}=3.002 \mathrm{MeV}$ corresponding to $2 n$ transfer and an effective $Q_{t r}$ value $=5.133 \mathrm{MeV}$ (corresponding to $2 n+4 n$ transfer). In principle, more than one transfer channel should be included in the couplings since multiple transfer channels could affect the observed cross sections. However, currently only one transfer channel can be included in CCFULL. Hence, two different calculations were done for the two different $Q$ values. The form factor, $F_{t}$, defined as the coupling strength, for transfer coupling in CCFULL is that for a pair transfer. The $F_{t}$ value was varied to get the best reproduction of the experimental data from which the final values of $0.3 \mathrm{MeV}$ and $0.35 \mathrm{MeV}$, respectively, for the two transfer $Q$ values were determined. Keeping the same values of $V_{o}$ and $a_{o}$, as those used in the calculations without coupling and with inelastic coupling (as given in Table III), the value of $r_{o}$ was varied to account for the renormalization in the barrier energy caused by inclusion of transfer couplings.

For the purpose of comparison, using exactly the same coupling schemes and the same $V_{o}$ as those used in the calculations using $a_{o}=0.89 \mathrm{fm}, \mathrm{CC}$ calculations were done for $a_{o}=0.65 \mathrm{fm}$, where only $r_{o}$ was varied to reproduce the average barrier as that obtained from the larger diffuseness calculations. No fits to the experimental fusion cross sections were done for these calculations. From Fig. 7, it is seen that the CC results for the inelastic and transfer couplings (dot-dashed line for $Q_{t r}=3.002 \mathrm{MeV}$ and solid line for $Q_{t r}=5.133 \mathrm{MeV}$ ) are very similar at above barrier energies with visible difference seen only at sub-barrier energies. The $l$ distributions corresponding to the different coupling schemes are given in Fig. 13 in Appendix A and behave as expected with increasing coupling resulting in a higher angular momentum cutoff.

$$
\text { 3. }{ }^{40} \mathrm{Ca}+{ }^{124} \mathrm{Sn}
$$

For this system, experimental fusion evaporation cross sections $\left(\sigma_{E R}\right)$, plotted as filled circles in Fig. 8, were taken from Refs. [26-28]. In order to compare the CC results with the experimental fusion cross sections, the fission cross sections obtained in the present measurement have been interpolated to the energy values corresponding to the experimental $\sigma_{E R}$. The sum of $\sigma_{E R}$ and these interpolated fission cross sections are plotted as unfilled squares in Figs. 8(a), 8(b), 8(d), and 8(e).

As in the case of the previous two systems, couplings to only the projectile and target excited states, namely, the $3^{-}$vibrational state in ${ }^{40} \mathrm{Ca}$ and the $2^{+}$and $3^{-}$vibrational states of ${ }^{124} \mathrm{Sn}$ (details in Table IV) were included initially. Similar to ${ }^{28} \mathrm{Si}+{ }^{136} \mathrm{Ba}$, the $\mathrm{CC}$ results thus obtained do not reproduce the experimental data at sub-barrier energies (see dashed line in Fig. 8). This system too has positive $Q$ values for $n$ transfer and earlier works [26-28] have shown the importance of transfer in affecting fusion. Thus, three different transfer coupling schemes were included: $Q_{t r}=3 \mathrm{MeV}$, with optimum $F_{t}=0.5 \mathrm{MeV} ; Q_{t r}=5.41 \mathrm{MeV}$ corresponding to $2 n$ transfer with $F_{t}=0.7 \mathrm{MeV}$ and $Q_{t r}=9.5 \mathrm{MeV}$ corresponding to $4 n$ transfer with $F_{t}=0.8 \mathrm{MeV}$. The $r_{o}$ value in each transfer coupling scheme was tweaked to account for the

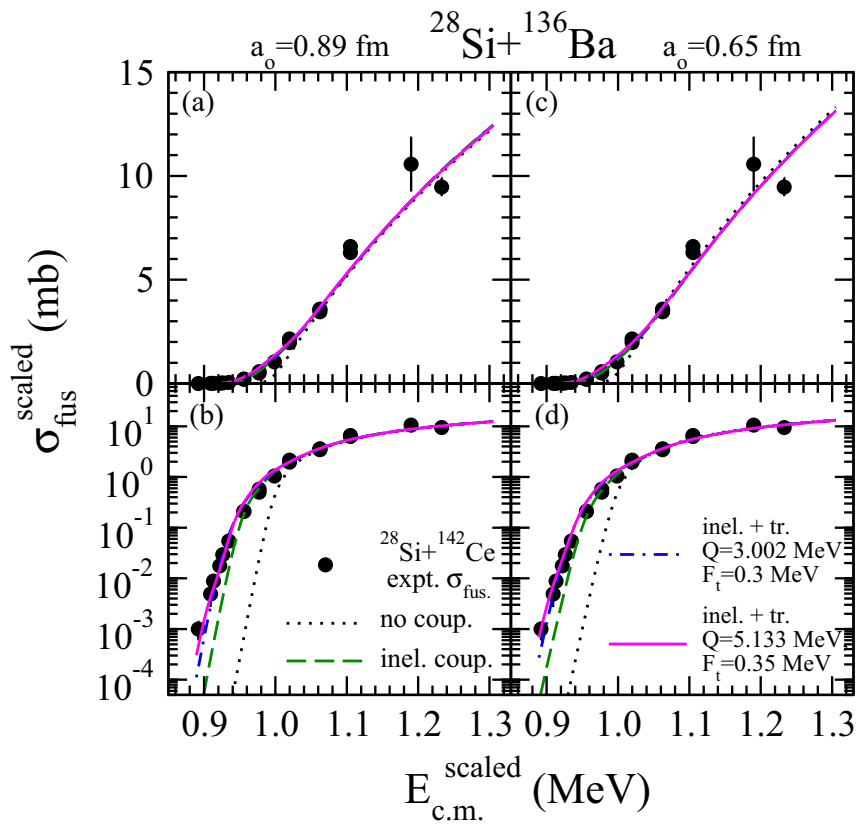

FIG. 7. Scaled experimental fusion cross sections (filled circles) for the ${ }^{28} \mathrm{Si}+{ }^{142} \mathrm{Ce}$ system taken from Ref. [24] plotted in (a), (c) linear and (b), (d) logarithmic scales. The results of the CC calculations with couplings to the target and projectile inelastic states are shown by the dashed lines. Results of additional transfer couplings with $Q_{t r}=3.002 \mathrm{MeV}$ and $F_{t}=0.3 \mathrm{MeV}$ are shown by the dot-dash line while using $Q_{t r}=5.133 \mathrm{MeV}$ and $F_{t}=0.35 \mathrm{MeV}$ are shown by the solid line. The left panel of the figure corresponds to calculations with $a_{o}=0.89 \mathrm{fm}$ while the right panel corresponds to $a_{o}=0.65 \mathrm{fm}$.

shift in the barrier energy introduced by the transfer coupling while keeping $V_{o}$ and $a_{o}$ values the same as that used in the no coupling and inelastic coupling schemes (values as given in Table III).

Referring to Fig. 8, CC results corresponding to inelastic and transfer coupling with $Q_{t r}=5.41 \mathrm{MeV}$ (solid line) gives the best reproduction of the fusion excitation function for the near and above barrier energy cross sections. However, at sub-barrier energies the agreement is poor. In contrast, results of the inelastic and transfer coupling with $Q_{t r}=3 \mathrm{MeV}$ (dotdashed line) gives a better reproduction of the fusion cross sections at below barrier energies with a poor fit above the barrier. The significant differences between the $\mathrm{CC}$ calculations with and without transfer couplings at the higher energies may be an artifact of the high transfer coupling strengths needed to reproduce the sub-barrier cross sections. It would be valuable, in the future, to explore the reasons for the behavior of both experiment and calculations at these energies for a detailed understanding of these observations.

The experimental fusion cross sections (unfilled squares) in the $E_{\text {c.m. }}$ range of $130-145 \mathrm{MeV}$, where the present experimental fission data have been taken, are not reproduced by the $\mathrm{CC}$ results using any of the coupling schemes for the larger diffuseness of $a_{o}=1.0 \mathrm{fm}$. Hence, the $l$ distribution used the in statistical model calculations described below, were taken from the $\mathrm{CC}$ results for an energy at which 


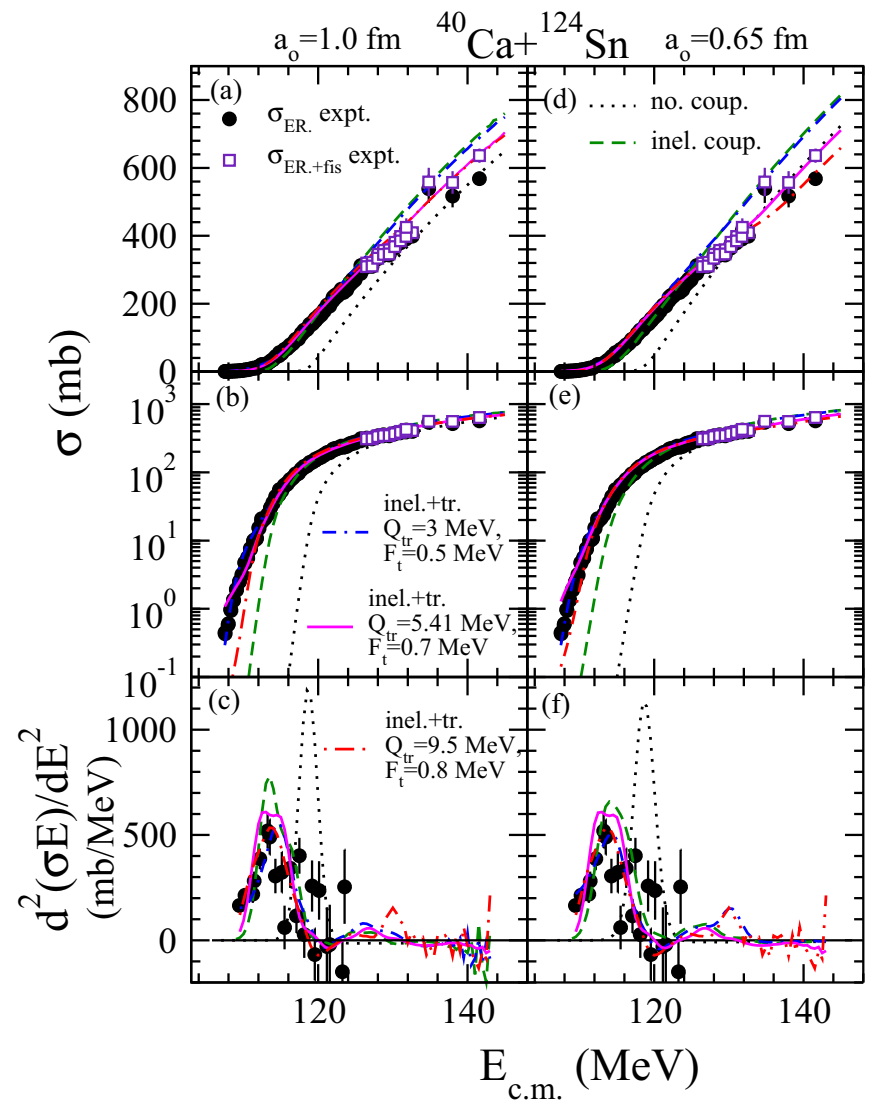

FIG. 8. Experimental fusion evaporation cross sections (filled circles) for the ${ }^{40} \mathrm{Ca}+{ }^{124} \mathrm{Sn}$ system taken from Refs. [26-28] and the sum of the fusion evaporation and interpolated fission cross sections (unfilled squares) plotted in the (a), (d) linear and (b), (e) logarithmic scale. Filled circles in (c), (f) show the barrier distribution extracted from the experimental cross sections. The dotted line corresponds to model calculations without any coupling whereas the dashed line are those obtained by coupling the projectile and target inelastic states (see text for details). The CC results obtained from projectile and target inelastic + transfer coupling with $Q_{t r}=3 \mathrm{MeV}$ are plotted as a dot-dash-dash line, with $Q_{t r}=5.41 \mathrm{MeV}$ as a solid line and with $Q_{t r}=9.5 \mathrm{MeV}$ as a dot-dashed line. The left panel of the figure shows the results for $a_{o}=1.0 \mathrm{fm}$ while the right panel shows those for $a_{o}=0.65 \mathrm{fm}$.

the predicted fusion cross sections matched the experimental fusion cross sections. For example, the experimental cross section at $E_{\text {c.m. }}=144.07 \mathrm{MeV}$ is $692 \mathrm{mb}$ but the CC results obtained using inelastic coupling scheme at this energy for $a_{o}=1.0 \mathrm{fm}$ gives a cross section of $744 \mathrm{mb}$. However, $\mathrm{CC}$ results corresponding to $E_{\mathrm{c} . \mathrm{m} .}=141.17 \mathrm{MeV}$ gives the matching cross section of $692 \mathrm{mb}$. Thus, the $l$ distribution for $141.17 \mathrm{MeV}$ was used in the statistical model calculations. The same procedure was done for all other energies and different coupling schemes. The different $l$ distributions thus obtained are plotted in Fig. 14 in Appendix A. Using the $l$ distributions calculated at the lower energies to match the experimental fusion cross sections means that the distributions do not extend to high $l$ values. The influence of the high $l$ tail on the fission cross sections predicted by the statistical model calculations is discussed in Sec. IV A. The $l$ distributions used in the statistical model calculations for $a_{o}=0.65 \mathrm{fm}$ are for the energies at which the fission measurements were made in the present work.

\section{STATISTICAL MODEL CALCULATIONS}

In order to introduce the concept of using fission cross sections to probe the fusion process at high angular momenta, a brief discussion of illustrative calculations carried out using $l$ distributions corresponding to two different diffuseness parameters, $a_{o}=0.65$ and $1.0 \mathrm{fm}$, is given in Sec. IV A below, followed by results of the actual comparison between the experimental fission cross sections with predictions of the statistical model calculations in Sec. IV B. We note here that for all calculations described below, the statistical model parameters are fixed first, and only then are the comparisons carried out to understand the changes in the fission crosssection predictions caused by the $l$ distributions.

\section{A. Concept}

The ${ }^{16} \mathrm{O}+{ }^{148} \mathrm{Sm}$ system is chosen to illustrate the concept. For this system, the CC calculations with the couplings as described in Sec. III B 1 were used to generate the $l$ distributions at the three energies $E_{\text {c.m. }}=82.04,90.21$, and $99.24 \mathrm{MeV}$ (see Table II) for which the fission cross sections have been precisely measured in this work. The CC calculations were done for two diffusenesses, $a_{o}=0.65 \mathrm{fm}$ and $1.0 \mathrm{fm}$ and the resulting $l$ distributions are plotted in Fig. 9(a) by solid and dashed lines, respectively, at the representative energy of $E_{\text {c.m. }}=99.24 \mathrm{MeV}$. Next, the whole $l$ distribution for $a_{o}=$ $0.65 \mathrm{fm}$ was scaled down by a constant factor [as shown by the dot-dot-dashed line in Fig. 9(a)] such that the resultant fusion cross section (i.e., $\sum_{l} \sigma_{l}$ ) matched that obtained from $\mathrm{CC}$ calculations with $a_{o}=1.0 \mathrm{fm}$. For the three energies, this scaling factor was $0.91,0.90$, and 0.88 , respectively. These $l$ distributions, corresponding to the two different diffuseness parameters of $a_{o}=0.65 \mathrm{fm}$ and $1.0 \mathrm{fm}$ as well as that for the scaled $a_{o}=0.65 \mathrm{fm}$, for the three energies were given as the input distributions to the statistical model code PACE [29]. Fission cross sections were calculated using the statistical model parameters given in Appendix B. The scaling factor, $k$, for the Sierk fission barrier [33] was fixed at 1 for these calculations. The PACE predictions for the three energies are plotted in Fig. 9(b) by filled circles for $a_{o}=0.65 \mathrm{fm}$, filled squares for $a_{o}=1.0 \mathrm{fm}$ and unfilled diamonds for the scaled $a_{o}=0.65 \mathrm{fm}$.

The following points are highlighted by these calculations:

(i) A large diffuseness parameter, $a_{o}=1.0 \mathrm{fm}$, has the effect of reducing the fusion cross sections substantially and this, in turn, reduces the fission cross sections by a much larger factor. For example, for $E_{\text {c.m. }}=99.24 \mathrm{MeV}, \sigma_{\text {fus }}=1487 \mathrm{mb}$ when $a_{o}=$ $0.65 \mathrm{fm}$ is used in the CC calculations, while $\sigma_{\text {fus }}=$ $1310 \mathrm{mb}$ for $a_{o}=1.0 \mathrm{fm}, \sim 12 \%$ less than the former. The fission cross sections obtained by inputting the $l$ distributions corresponding to these two different diffusenesses [Fig. 9(a)] in PACE are $\sigma_{\text {fis }}=36 \mathrm{mb}$ for 

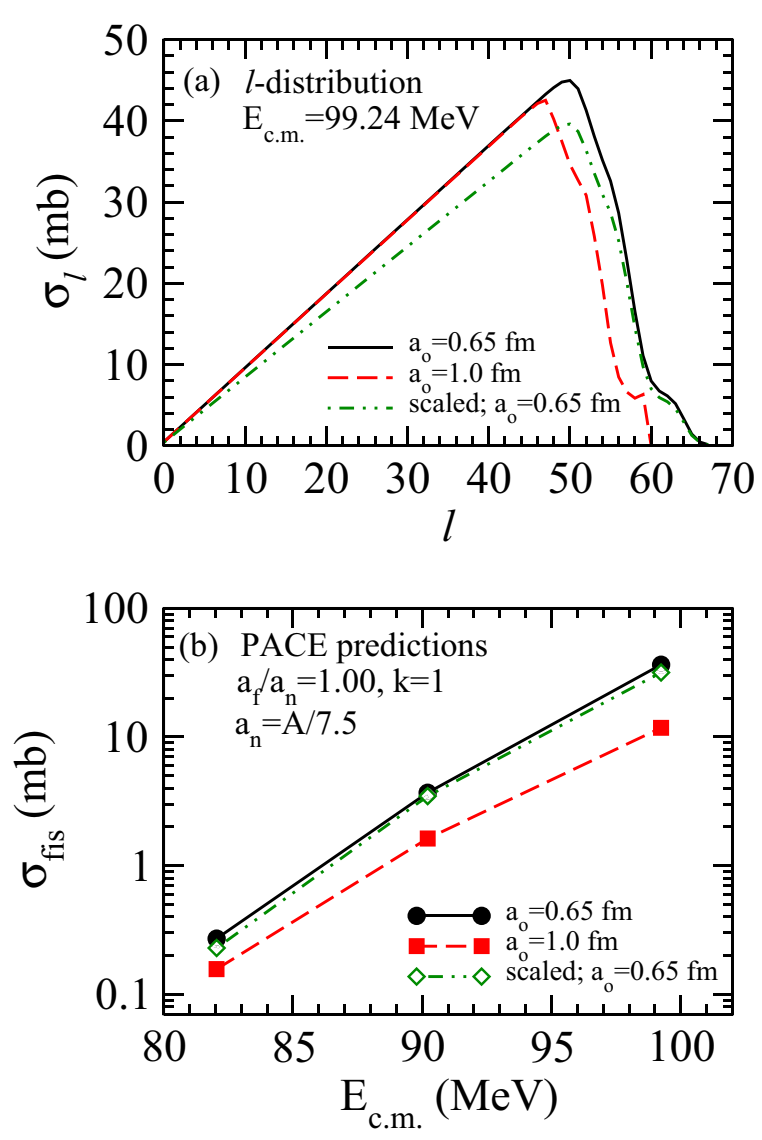

FIG. 9. (a) $l$ distribution for the ${ }^{16} \mathrm{O}+{ }^{148} \mathrm{Sm}$ system at the representative energy of $E_{\text {c.m. }}=99.24 \mathrm{MeV}$. The solid line is the $l$ distribution obtained from CC calculations for the smaller diffuseness value $\left(a_{o}=0.65 \mathrm{fm}\right)$ of the nuclear potential, while the dashed line corresponds to the one for the larger diffuseness $\left(a_{o}=1.0 \mathrm{fm}\right)$. The dot-dot-dashed line is obtained by scaling the $l$ distribution for $a_{o}=0.65 \mathrm{fm}$ by 0.88 such that partial cross sections summed over all $l$ values is the same as that corresponding to $a_{o}=1.0 \mathrm{fm}$. (b) shows the fission cross section predictions from PACE when the three distributions shown in (a) are given as its input distribution.

$a_{o}=0.65 \mathrm{fm}$ and $\sigma_{\mathrm{fis}}=12 \mathrm{mb}$ for $a_{o}=1.0 \mathrm{fm}$, three times less than the former. For the other two energies, the predicted fission cross sections for $a_{o}=1.0 \mathrm{fm}$ are $\sim 2$ times less than those for $a_{o}=0.65 \mathrm{fm}$. From these, it is clear that small changes in the fusion cross sections cause a huge change in the resultant fission cross-section predictions, due to the high $l$ tail of the distribution extending to different angular momentum values. In other words, fission cross sections amplify the small changes in the fusion cross sections. This observation is a compelling motivation for further investigations of using fission cross sections as a probe to understand the fusion process at high angular momentum.

(ii) For $E_{\text {c.m. }}=99.24 \mathrm{MeV}$, the fission cross section resulting from the scaled down $a_{o}=0.65 \mathrm{fm} l$ distribution is $\sigma_{\text {fis }}=32 \mathrm{mb}$, which is less by $\sim 11 \%$ as compared to the unscaled $a_{o}=0.65 \mathrm{fm}$ results, very close to the scaling factor (i.e., scaled down by $\sim 12 \%$ ) used above for matching the fusion cross sections. We note here that the reason for the $11 \%$ reduction (and not 12\%) in fission predictions corresponding to the scaled $a_{o}=0.65 \mathrm{fm}$ cross sections is only due to the statistics of fission events. The probability of fission in both the cases at this energy remains the same and is equal to 0.024 and thus the results are consistent. A similar observation is found for the other two energies as well. This shows that the fission cross sections reduce by the same amount by which the $l$ distributions were scaled for matching the fusion cross sections.

(iii) The calculations presented above indicate why highquality fusion cross-section measurements are required. If the fusion cross sections have an error between $\sim 9 \%-12 \%$, then the predicted fission cross sections would differ by a factor of $\sim 2-3$. Keeping these concepts in mind, a comparison of the experimental fission cross sections with the predictions from statistical model calculations is presented in the section below.

\section{B. Comparison of experimental fission cross sections with statistical model predictions}

Following the procedure described in the illustrative calculations in Sec. IV A, the $l$ distributions, corresponding to $a_{o}=$ $0.65 \mathrm{fm}$ and $a_{o}>0.65 \mathrm{fm}$, obtained from the CC calculations for each of the three systems (plotted in Figs. 6, 13, and 14) were used in PACE to calculate the fission cross sections for each system. The statistical model parameters used, except for the scaling factor, $k$, for the Sierk fission barrier [33], are given in Appendix B. The ${ }^{16} \mathrm{O}+{ }^{148} \mathrm{Sm}$ system was taken as a reference system to fix $k$. This was done by using $a_{f} / a_{n}=$ 1.00 and varying $k$ in the PACE calculations to get a minimum $\chi^{2}$ fit to the experimental fission cross sections for the system. The best fit $k$ values of 0.994 for $a_{o}=0.99 \mathrm{fm}$ and 1.084 for $a_{o}=0.65 \mathrm{fm}$ were thus obtained. The best fit $k$ values are different as the $l$ distributions, and hence the predicted fission cross sections, are diffuseness dependent. A similar procedure was done taking $a_{f} / a_{n}=1.04$. Though different values of $k$ were obtained, the calculated fission cross sections were very similar. Thus, in the following, for simplicity, comparisons are shown only for $a_{f} / a_{n}=1.00$.

The predictions of the statistical model calculations for the ${ }^{16} \mathrm{O}+{ }^{148} \mathrm{Sm}$ system are plotted in Fig. 10. The predictions corresponding to the larger diffuseness of $a_{o}=0.99 \mathrm{fm}$ (dot-dashed line) give a better fit to the experimental fission cross sections as compared to those corresponding to $a_{o}=$ $0.65 \mathrm{fm}$. This can also be seen from Figs. 11(a) and 11(b) where the ratio of the fission predictions from PACE to the experimental fission cross sections has been plotted. From this figure, it can be clearly seen that the fission predictions for the larger diffuseness gives a much better reproduction of the experimental cross sections, with the ratios deviating less from 1.0 [Fig. 11(a)] than the results for $a_{o}=0.65 \mathrm{fm}$ [Fig. 11(b)]. This can be understood from the differences in the $l$ distributions for $a_{o}=0.99 \mathrm{fm}$ and $a_{o}=0.65 \mathrm{fm}$ as shown in Figs. 6(b) and 6(d). These visible differences in the 


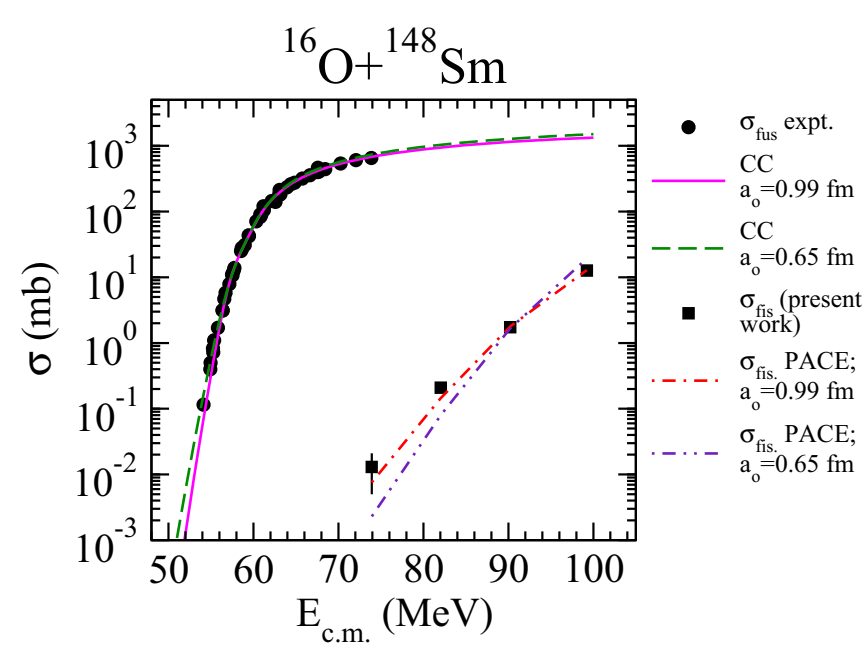

FIG. 10. Comparison of the experimental fusion [18-21] (filled circles) and fission (filled squares) cross sections from the present work with the predictions of the statistical model calculations using PACE. The solid and dashed line are the $\mathrm{CC}$ results obtained by coupling the inelastic states in the projectile and target and static deformations in the target (same inelastic coupling results as plotted in Fig. 5 by solid and dot-dash lines) for the two different diffuseness parameters, respectively. The corresponding fission cross sections estimated from PACE are plotted as dot-dashed line for $a_{o}=0.99 \mathrm{fm}$ and dot-dot-dashed lines for $a_{o}=0.65 \mathrm{fm}$.

fission cross section predictions point to the importance of the shape of the $l$ distribution, especially its tail, in determining the fission cross-section predictions.

According to Bohr's independence hypothesis, for the same compound nucleus formed via different entrance channels, its decay should be independent of the way it was formed and hence the same fission parameters as those used in ${ }^{16} \mathrm{O}+{ }^{148} \mathrm{Sm}$ calculations should be valid. Thus, taking the same values of $k$ as those obtained for ${ }^{16} \mathrm{O}+{ }^{148} \mathrm{Sm}$ system, PACE calculations for the ${ }^{28} \mathrm{Si}+{ }^{136} \mathrm{Ba}$ and ${ }^{40} \mathrm{Ca}+{ }^{124} \mathrm{Sn}$ systems were carried out. Figure 12 shows the results obtained for the ${ }^{28} \mathrm{Si}+{ }^{136} \mathrm{Ba}$ system. We note here that just like the fusion cross section, the fission cross sections for the system have been scaled using the same scaling factors mentioned in Sec. III B 2, i.e., 0.0149 and 0.0104 , respectively, for the cross sections and energy. From the comparisons for the inelastic coupling scheme [Fig. 12(a)], it can be seen that the statistical model calculations underpredict the experimental fission cross sections, especially at the lower two energies, even though the experimental fusion cross sections are satisfactorily reproduced by the calculations in this energy region. This is also reflected by the larger deviations from 1.0 [filled circles in Figs. 11(c) and 11(d)] at these energies. The apparent agreement with fusion cross sections, but significant disagreement with fission cross sections shows that the calculated angular momentum distributions do not extend as high in angular momentum, as would be required to match the experimental fission cross sections. This sensitivity of fission to higher partial waves is consistent with the illustrative example of Sec. IV. Predictions corresponding to inelastic and transfer coupling give a much better reproduction at all four ener-
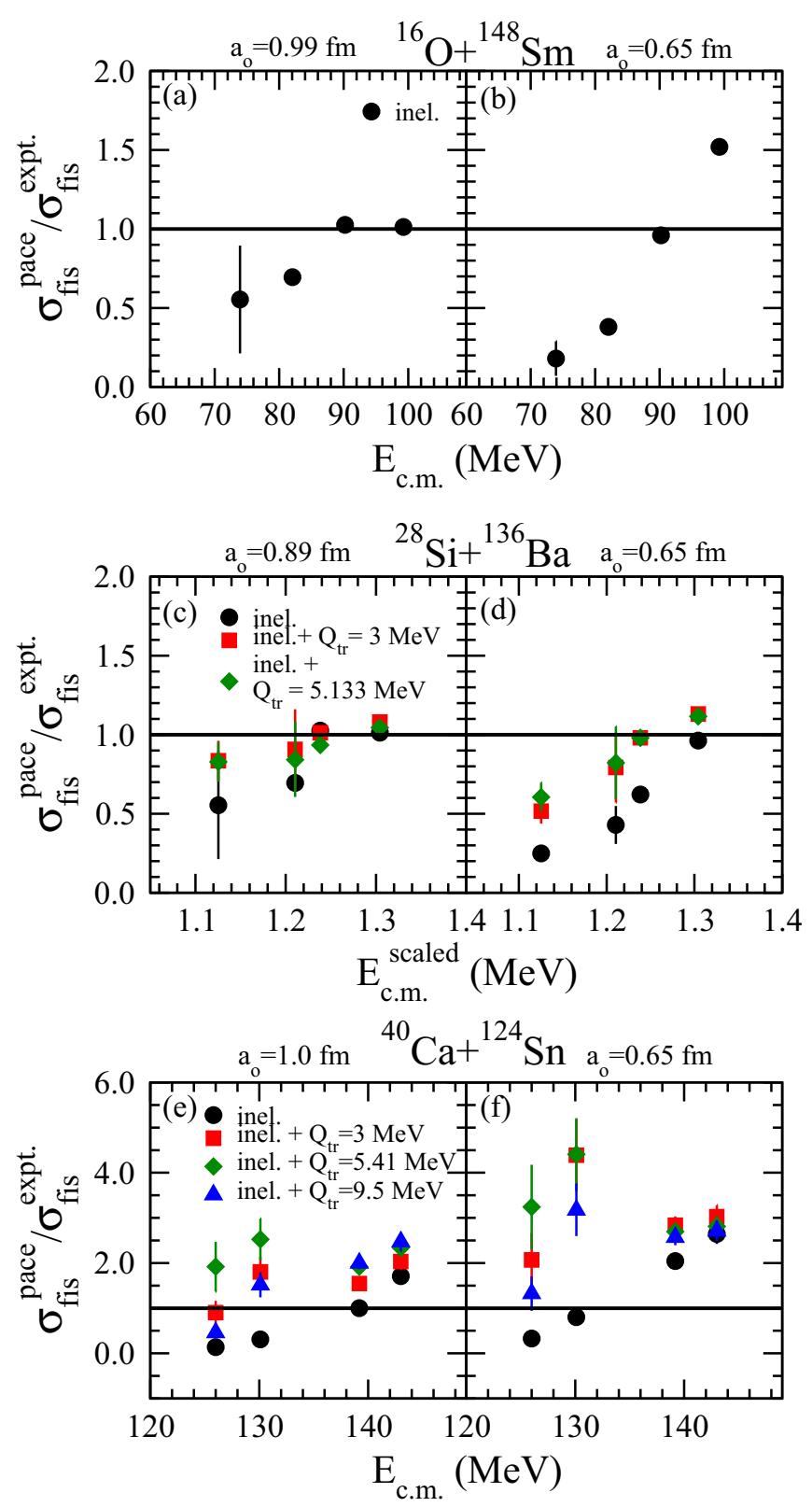

FIG. 11. Ratio of the fission cross section predictions from PACE to the experimental fission cross sections measured in this work as a function of center-of-mass energy for (a), (b) ${ }^{16} \mathrm{O}+{ }^{148} \mathrm{Sm}$, (c), (d) ${ }^{28} \mathrm{Si}+{ }^{136} \mathrm{Ba}$, and (e), (f) ${ }^{40} \mathrm{Ca}+{ }^{124} \mathrm{Sn}$ systems. The left side plots are for the larger diffuseness while the right side plots are for $a_{o}=0.65 \mathrm{fm}$. The horizontal solid line in each plot corresponds to a ratio of 1.0 .

gies. Best overall agreement between the PACE predictions and experimental fission cross sections are seen for inelastic and transfer with $Q_{t r}=3.002 \mathrm{MeV}$ coupling scheme using $a_{o}=0.89 \mathrm{fm}$ [dot-dashed line in Fig. 12(b) and filled squares in Fig. 11(c)]. Coupling to a transfer channel in addition to the inelastic channel extends the $l$ distribution to larger angular momentum values as compared to when only inelastic coupling is included in the calculations, as can be seen from Figs. 14(c), 14(g), 14(d), and 14(h) in Appendix A. This leads to larger fission cross-section predictions. For inelastic and 


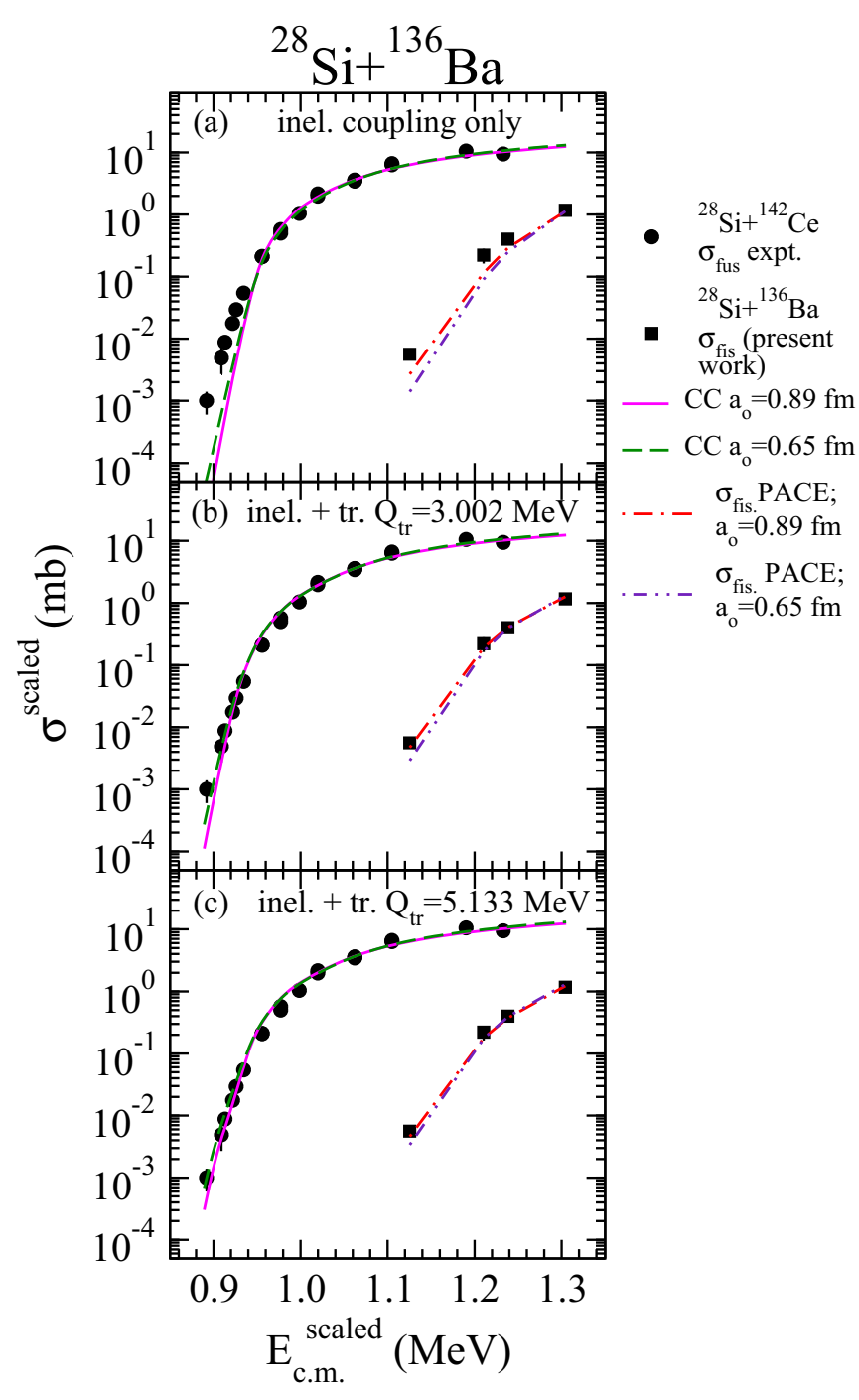

FIG. 12. Similar to Fig. 10 but for ${ }^{28} \mathrm{Si}+{ }^{136} \mathrm{Ba}$ system. Here, the scaled cross sections have been plotted in order to show the comparison between experimental fusion cross sections for ${ }^{28} \mathrm{Si}+$ ${ }^{142} \mathrm{Ce}$ [24] (filled circles) with predictions of model calculations for the ${ }^{28} \mathrm{Si}+{ }^{136} \mathrm{Ba}$ system. In (a) are plotted the results obtained from PACE when $l$ distributions extracted from inelastic coupling only are used in the calculations. The solid and dashed lines are the same as the dashed line in Fig. 7 for the two different diffusenesses. Similarly, (b) shows the results for inelastic + transfer with $Q_{t r}=3.002 \mathrm{MeV}$ while (c) shows the results for inelastic + transfer with $Q_{t r}=5.133 \mathrm{MeV}$ with the solid and dashed lines the same as the dot-dashed and solid line in Fig. 7 respectively for the two diffuseness and coupling schemes. The dot-dashed lines are the fission cross section predictions obtained from PACE by using $a_{o}=0.89 \mathrm{fm} l$ distributions while the dot-dot-dashed lines are those using $a_{o}=0.65 \mathrm{fm}$ distributions.

transfer with $Q_{t r}=5.133 \mathrm{MeV}$, small changes in the shape of the tail region in the $l$ distribution are observed [Figs. 14(d) and $14(\mathrm{~h})]$ and the agreement between the experimental and calculated values is observed to degrade.

Results for the ${ }^{40} \mathrm{Ca}+{ }^{124} \mathrm{Sn}$ system are plotted in Fig. 13 while the ratio of the fission cross section predicted by PACE to the experimental fission cross sections are plotted

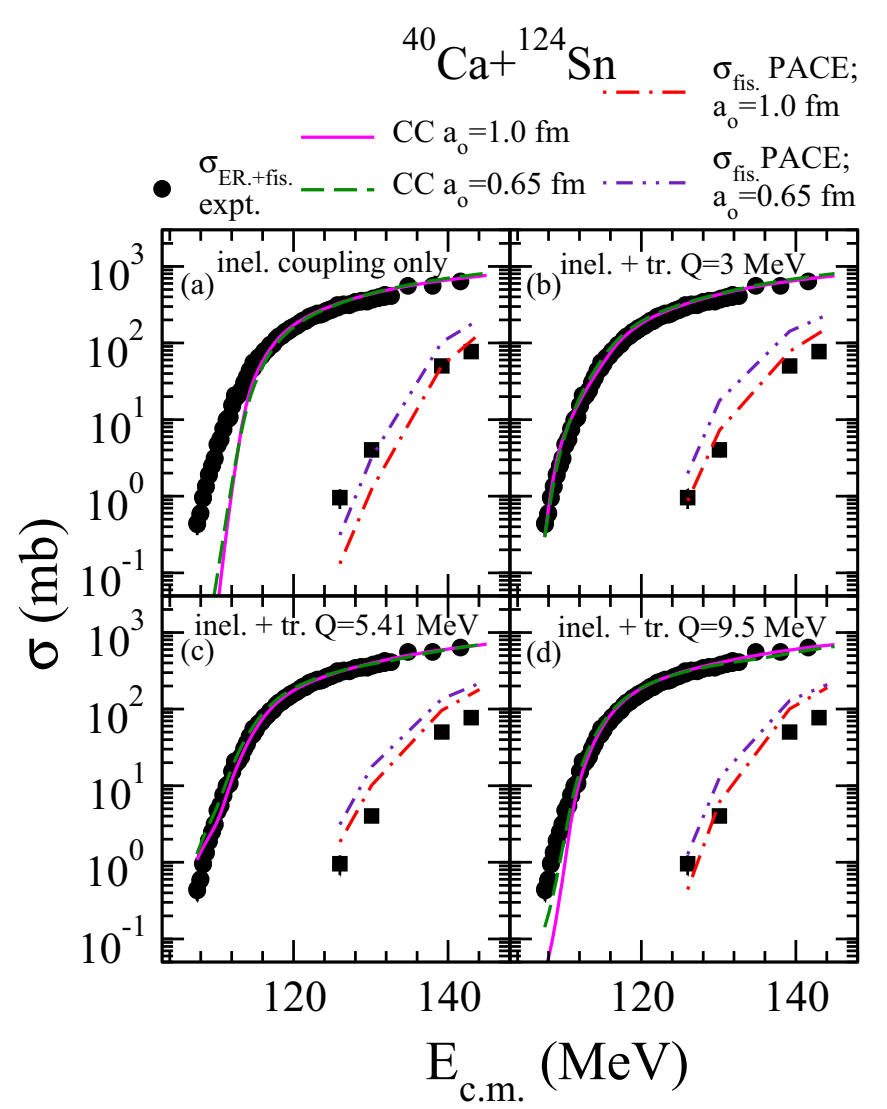

FIG. 13. Similar to Fig. 12, but for ${ }^{40} \mathrm{Ca}+{ }^{124} \mathrm{Sn}$ system. Results from PACE corresponding to $l$ distributions obtained from inelastic coupling only are plotted in (a) whereas those corresponding to results from inelastic + transfer coupling are plotted in (b) for transfer $Q_{t r}=3 \mathrm{MeV}$, in (c) for $Q_{t r}=5.41 \mathrm{MeV}$, and in (d) for $Q_{t r}=9.5 \mathrm{MeV}$. The solid and dashed lines representing the CC results are the same as the $\mathrm{CC}$ results shown in Fig. 8 for the respective coupling scheme and the two different diffusenesses. The dot-dashed lines are the fission cross-section predictions from PACE corresponding to $l$ distributions obtained from the CC calculations using $a_{o}=1.0 \mathrm{fm}$ and dot-dot-dashed lines are those corresponding to $a_{o}=0.65 \mathrm{fm}$.

in Figs. 11(e) and 11(f) for the diffuseness parameters of $a_{o}=1.0 \mathrm{fm}$ and $a_{o}=0.65 \mathrm{fm}$ respectively. As for the other two systems, fission cross section predictions for this system corresponding to the larger diffuseness parameter of $a_{o}=$ $1.0 \mathrm{fm}$ gives an overall better agreement with the respective experimental cross sections. For the inelastic coupling results [Figs. 13(a) and 11(e)], reasonable agreement only at the higher two energies is observed. As in the case of ${ }^{28} \mathrm{Si}+$ ${ }^{136} \mathrm{Ba}$ system, inclusion of transfer coupling (inelastic and transfer with $Q_{t r}=3 \mathrm{MeV}$ ) leads to an overall improved agreement between the experimental fission cross sections and the respective calculated values at all four energies. Coupling to transfer channel with $Q_{t r}>3 \mathrm{MeV}$ overpredicts the fission cross sections, especially at the higher energies. The statistical model predictions for ${ }^{40} \mathrm{Ca}+{ }^{124} \mathrm{Sn}$ show a stronger dependence on the $Q$ value used in the transfer couplings as compared to that observed for the ${ }^{28} \mathrm{Si}+{ }^{136} \mathrm{Ba}$ system. 


\section{SUMMARY AND CONCLUSIONS}

To summarize, the present work aimed to investigate the use of fission cross sections as a sensitive probe of fusion dynamics at high angular momentum. Fission of the $\mathrm{CN}{ }^{164} \mathrm{Yb}$ populated by three different entrance channels, ${ }^{16} \mathrm{O}+{ }^{148} \mathrm{Sm}$, ${ }^{28} \mathrm{Si}+{ }^{136} \mathrm{Ba}$, and ${ }^{40} \mathrm{Ca}+{ }^{124} \mathrm{Sn}$ has been measured for this purpose. Fission fragment angular distributions at four energies, each above their respective Coulomb barriers, have been measured for the three systems and the angle integrated fission cross sections extracted. These along with the experimental fusion cross sections available from literature have been used to constrain the statistical model fission calculations. The sensitivity of the results to different coupling schemes was investigated.

Fission cross sections are shown to be very sensitive to the shape of the angular momentum distributions as illustrated by calculations, where a large change in the fission predictions is seen for a small change in the fusion cross sections for different diffuseness and thus different $l$ distributions used for the calculations. Comparison of the experimental and calculated fission cross sections for the three systems studied shows that an overall better reproduction of the experimental fission

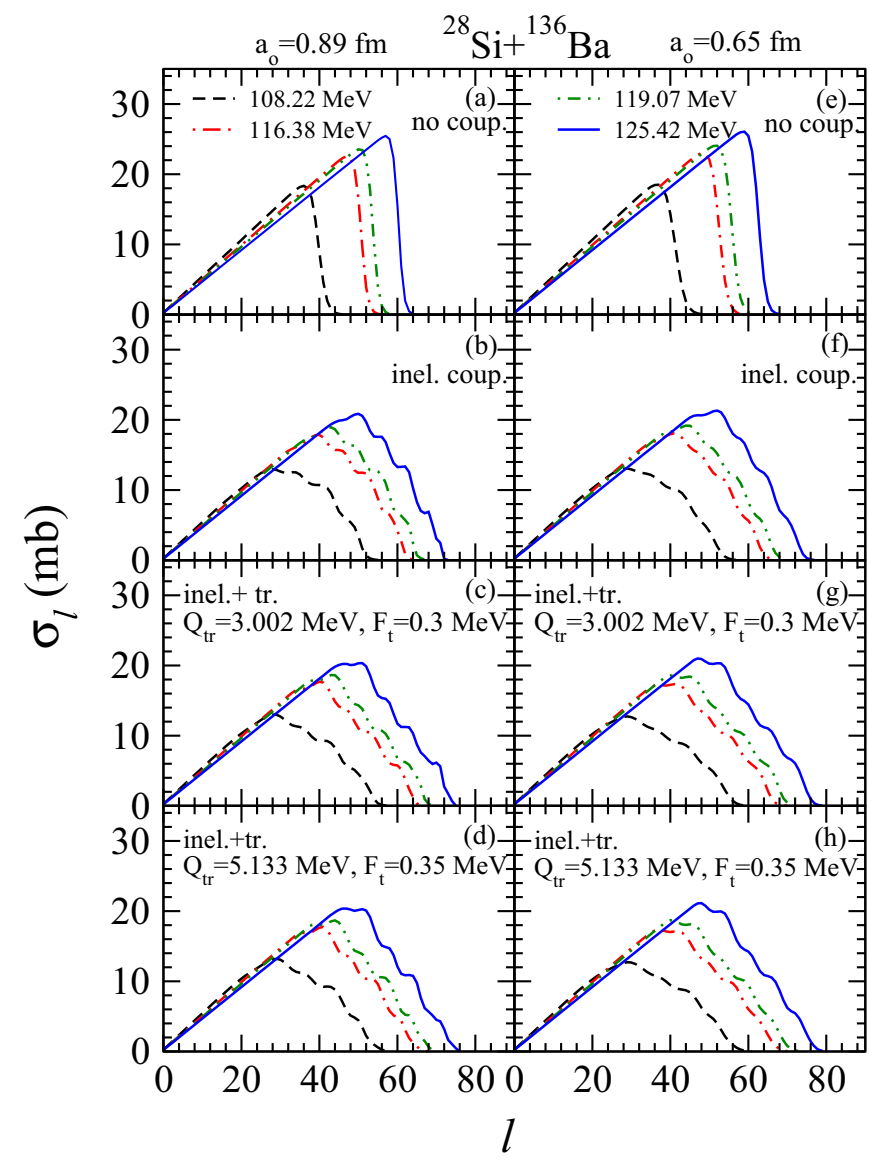

FIG. 14. $l$ distributions obtained from calculations for the ${ }^{28} \mathrm{Si}+$ ${ }^{136} \mathrm{Ba}$ system (a), (e) without any coupling, (b), (f) inelastic coupling only and (c), (d), (g), and (h) inelastic + transfer coupling, for the two different diffuseness parameters. cross sections is observed only for the larger diffuseness even though the experimental fusion cross sections are fairly well reproduced by the calculations corresponding to both $a_{o} \gg 0.65 \mathrm{fm}$ and $a_{o}=0.65 \mathrm{fm}$ at these energies. In addition, the fission cross sections for all systems are also shown to be much more sensitive to the coupling schemes used as compared to the fusion cross sections. Both these observations indicate how an amplified effect of small changes in the angular momentum distribution can be seen by using fission cross sections as probes. The results of this work thus point to how a simultaneous description of fusion and fission is advantageous for developing a better understanding of fusion at high angular momenta. Results from this work also indicate that measurement of precise fusion and well as fission cross sections is necessary as the results can drastically change as a result of cross-section uncertainties.

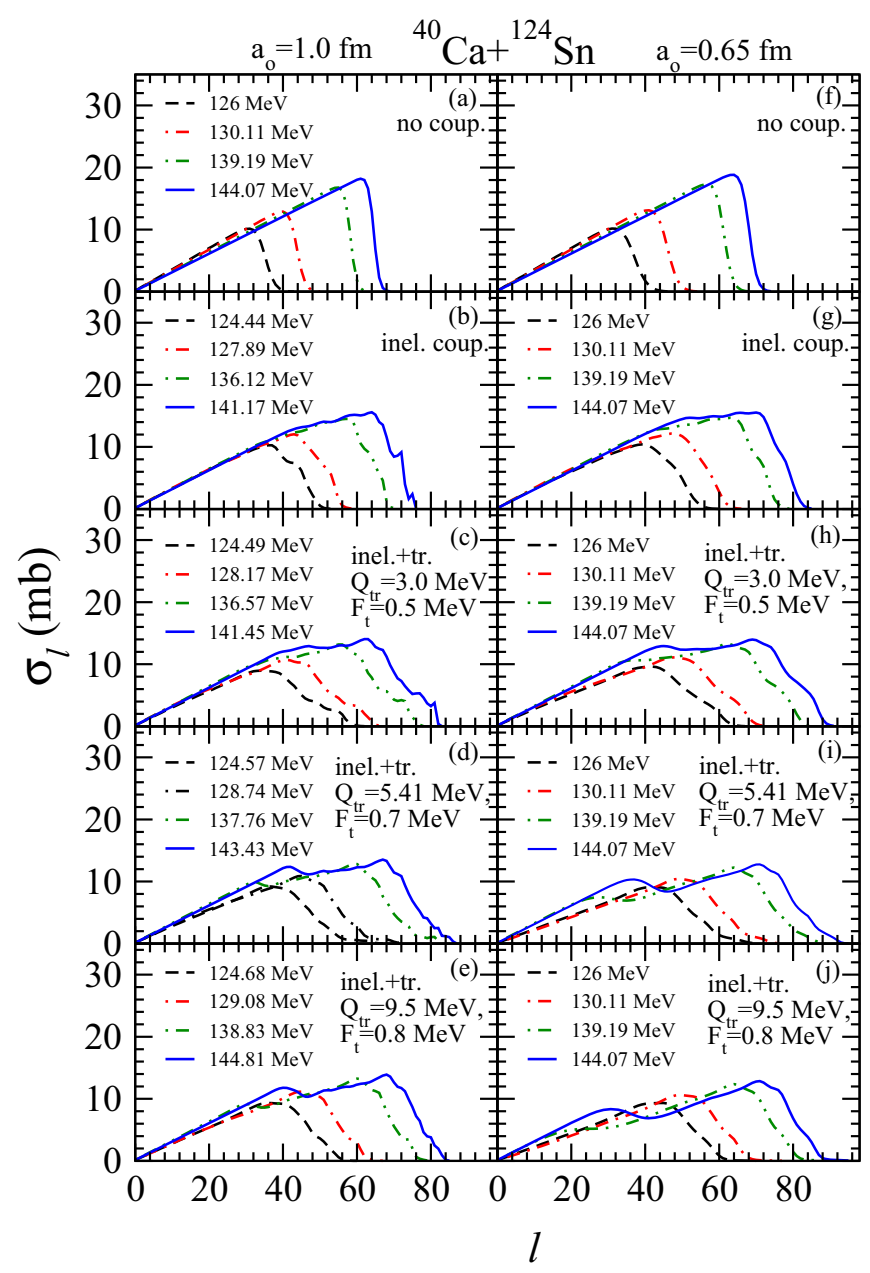

FIG. 15. $l$ distribution obtained from calculations for the ${ }^{40} \mathrm{Ca}+$ ${ }^{124} \mathrm{Sn}$ system. (a), (f) shows the distributions obtained without any coupling, (b), (g) are obtained from projectile + target inelastic coupling only while those obtained from projectile + target inelastic + transfer coupling with different $Q_{t r}$ values are plotted in (c), (h) for $Q_{t r}=3 \mathrm{MeV}$, (d), (i) for $Q_{t r}=5.41 \mathrm{MeV}$ and (e), (j) for $Q_{t r}=9.5 \mathrm{MeV}$, for the two diffuseness parameters. 


\section{ACKNOWLEDGMENTS}

The authors thank the accelerator and technical staff of the Heavy Ion Accelerator Facility at the Australian National University for their help during the experiment. The support from Australian Research Council through Grants No. DE140100784, No. DP160101254, No. FL110100098, and No. FT120100760 and support for the HIAF accelerator operations from the Australian Federal Government NCRIS program is gratefully acknowledged.

\section{APPENDIX A: $l$ DISTRIBUTIONS FOR ${ }^{28} \mathrm{Si}+{ }^{136} \mathrm{Ba}$ AND ${ }^{40} \mathrm{Ca}+{ }^{124} \mathrm{Sn}$ SYSTEMS}

The $l$ distributions obtained from CCFULL with different coupling schemes described in Secs. III B 2 and III B 3 for
${ }^{28} \mathrm{Si}+{ }^{136} \mathrm{Ba}$ and ${ }^{40} \mathrm{Ca}+{ }^{124} \mathrm{Sn}$ systems are plotted in Figs. 14 and 15 , respectively.

\section{APPENDIX B: STATISTICAL MODEL PARAMETERS}

The statistical model parameters in PACE were fixed as follows: The level density parameter at equilibrium deformation, $a_{n}$, for all calculations was taken as $a_{n}=A / 7.5$ following the semiempirical formula given in Eq. (A.9) of Ref. [30]. Determination of $F_{2}$ in the equation requires the values of fissility $(x)$ and $y=1-x$. The fissility was calculated to be $x=0.5872$ according to the relation given by Eq. (2) of Ref. [31] and $y=0.4128$. Using these, the value of $F_{2}$ was determined to be $F_{2}=1.284$ from Table III of Ref. [32]. The ratio $a_{f} / a_{n}$, where $a_{f}$ is the level density parameter at the saddle point, was taken as 1.00 .
[1] P. Armbruster, Annu. Rev. Nucl. Part. Sci. 50, 411 (2000).

[2] D. J. Hinde and M. Dasgupta, Nucl. Phys. A 787, 176 (2007).

[3] J. O. Newton, R. D. Butt, M. Dasgupta, D. J. Hinde, I. I. Gontchar, C. R. Morton, and K. Hagino, Phys. Rev. C 70, 024605 (2004).

[4] R. O. Akyuz and A. Winther, in Proceedings of the International School of Physics Enrico Fermi, edited by R. A. Broglia, C. H. Dasso, and R. Richi (North-Holland, Amsterdam, 1981).

[5] L. R. Gasques, M. Evers, D. J. Hinde, M. Dasgupta, P.R.S. Gomes, R. M. Anjos, M. L. Brown, M. D. Rodríguez, R. G. Thomas, and K. Hagino, Phys. Rev. C 76, 024612 (2007).

[6] M. Evers, M. Dasgupta, D. J. Hinde, L. R. Gasques, M. L. Brown, R. Rafiei, and R. G. Thomas, Phys. Rev. C 78, 034614 (2008).

[7] D. J. Hinde, R. L. Ahlefeldt, R. G. Thomas, K. Hagino, M. L. Brown, M. Dasgupta, M. Evers, L. R. Gasques, and M. D. Rodríguez, Phys. Rev. C 76, 014617 (2007).

[8] M. Dasgupta, D. J. Hinde, J. O. Newton, and K. Hagino, Prog. Theo. Phys. Supp. 154, 209 (2004).

[9] S. Cohen, F. Plasil, and W. J. Świątecki, Ann. Phys. (NY) 82, 557 (1974).

[10] R. J. Charity, J. R. Leigh, J. J. M. Bokhorst, A. Chatterjee, G. S. Foote, D. J. Hinde, J. O. Newton, S. Ogaza, and D. Ward, Nucl. Phys. A 457, 441 (1986).

[11] Y. Kondo, B. A. Robson, J. J. M. Bokhorst, D. J. Hinde, and J. R. Leigh, Phys. Rev. C 35, 828 (1987).

[12] D. J. Hinde, M. Dasgupta, J. R. Leigh, J. C. Mein, C. R. Morton, J. O. Newton, and H. Timmers, Phys. Rev. C 53, 1290 (1996).

[13] V. E. Viola, K. Kwiatkowski, and M. Walker, Phys. Rev. C 31, 1550 (1985).

[14] B. B. Back, R. R. Betts, J. E. Gindler, B. D. Wilkins, S. Saini, M. B. Tsang, C. K. Gelbke, W. G. Lynch, M. A. McMahan, and P. A. Baisden, Phys. Rev. C 32, 195 (1985).

[15] R. du Rietz, E. Williams, D. J. Hinde, M. Dasgupta, M. Evers, C. J. Lin, D. H. Luong, C. Simenel, and A. Wakhle, Phys. Rev. C 88, 054618 (2013).

[16] K. Hagino, N. Rowley, and A. T. Kruppa, Comp. Phys. Comm. 123, 143 (1999).
[17] M. Dasgupta, D. J. Hinde, N. Rowley, and A. M. Stefanini, Annu. Rev. Nucl. Part. Sci. 48, 401 (1998).

[18] R. G. Stokstad, Y. Eisen, S. Kaplanis, D. Pelte, U. Smilansky, and I. Tserruya, Phys. Rev. Lett. 41, 465 (1978).

[19] R. G. Stokstad, Y. Eisen, S. Kaplanis, D. Pelte, U. Smilansky, and I. Tserruya, Phys. Rev. C 21, 2427 (1980).

[20] D. E. DiGregorio, M. diTada, D. Abriola, M. Elgue, A. Etchegoyen, M. C. Etchegoyen, J. O. Fernández Niello, A. M. J. Ferrero, S. Gil, A. O. Macchiavelli, A. J. Pacheco, J. E. Testoni, P. R. Silveira Gomes, V. R. Vanin, R. L. Neto, E. Crema, and R. G. Stokstad, Phys. Rev. C 39, 516 (1989).

[21] J. R. Leigh, M. Dasgupta, D. J. Hinde, J. C. Mein, C. R. Morton, R. C. Lemmon, J. P. Lestone, J. O. Newton, H. Timmers, J. X. Wei, and N. Rowley, Phys. Rev. C 52, 3151 (1995).

[22] S. Raman, C. W. Nestor, JR., and P. Tikkanen, At. Data Nucl. Data Tables 78, 1 (2001).

[23] T. Kibédi and R. H. Spear, At. Data Nucl. Data Tables 80, 35 (2002).

[24] S. Gil, F. Hasenbalg, J. E. Testoni, D. Abriola, M. C. Berisso, M. di Tada, A. Etchegoyen, J. O. Fernández Niello, A. J. Pacheco, A. Charlop, A. A. Sonzogni, and R. Vandenbosch, Phys. Rev. C 51, 1336 (1995).

[25] P.R.S. Gomes, J. Lubian, I. Padron, and R. M. Anjos, Phys. Rev. C 71, 017601 (2005).

[26] A. M. Stefanini, L. Corradi, D. Ackermann, C. J. Lin, L. F. Zheng, S. Beghini, G. Montagnoli, F. Scarlassara, and G. F. Segato, IL Nuovo Cimento 111, 895 (1998).

[27] F. Scarlassara, S. Beghini, G. Montagnoli, G. F. Segato, D. Ackermann, L. Corradi, C. J. Lin, A. M. Stefanini, and L. F. Zheng, Nucl. Phys. A 672, 99 (2000).

[28] J. J. Kolata, A. Roberts, A. M. Howard, D. Shapira, J. F. Liang, C. J. Gross, R. L. Varner, Z. Kohley, A. N. Villano, H. Amro, W. Loveland, and E. Chavez, Phys. Rev. C 85, 054603 (2012).

[29] A. Gavron, Phys. Rev. C 21, 230 (1980).

[30] J. Tōke and W. J. Świątecki, Nucl. Phys. A 372, 141 (1981).

[31] J. P. Blocki, H. Feldmeier, and W. J. Świątecki, Nucl. Phys. A 459, 145 (1986).

[32] W. D. Myers and W. J. Świątecki, Ann. Phys. (NY) 84, 186 (1974).

[33] A. J. Sierk, Phys. Rev. C 33, 2039 (1986). 